\title{
Time Versus Energy in the Averaged Optimal Coplanar Kepler Transfer towards Circular Orbits
}

\author{
Bernard Bonnard • Helen C. Henninger • \\ Jana Němcová · Jean-Baptiste Pomet
}

\begin{abstract}
This article makes a study of the averaged optimal coplanar transfer towards circular orbits. Our objective is to compare this problem when the cost minimized is transfer time to the same problem when the cost minimized is energy consumption. While the minimum energy case leads to the analysis of a $2 D-$ Riemannian metric using the standard tools of Riemannian geometry, the minimum time case is associated with a Finsler metric which is not smooth. Nevertheless a qualitative analysis of the geodesic flow is given in this article to describe the optimal transfers of the time minimal case.
\end{abstract}

Keywords Averaging, Optimal control, Low thrust orbit transfer, Geodesic convexity, Riemann-Finsler Geometry

\section{Introduction}

We consider the controlled Kepler equation describing orbital transfers with low thrust engines, that we normalize as

$$
\ddot{q}=-\frac{q}{\|q\|^{3}}+u
$$

the control is constrained by $\|u\| \leq \varepsilon$, where $\varepsilon$ is a small parameter. The phase space, or state space, is the one with coordinates $(q, \dot{q})$. Let $K=\frac{1}{2}\|\dot{q}\|^{2}-1 /\|q\|$ be the mechanical energy of the uncontrolled system and $X$ be the elliptic domain:

$$
X=\{K<0, q \wedge \dot{q} \neq 0\} .
$$

For the free motion $(u=0)$, the solutions that lie in $X$ are ellipses - or more precisely closed curves that project on the $q$ component as ellipses - and they form a foliation of $X$.

In this domain, we may chose coordinates $(x, l)$ where $x$ is made of independent first integrals of the uncontrolled motion (so that $x$ describes the geometry of the ellipses) and the "longitude" $l$ defines the position of the

The second author was partially supported by Thales Alenia Space and région Provence Alpes Côte d'Azur

B. Bonnard

Institut de Mathématiques de Bourgogne, Université de Bourgogne,

9 avenue Alain Savary, 21078 Dijon, France.

E-mail: bernard.bonnard@u-bourgogne.fr

On leave to: team McTAO, Inria Sophia Antipolis Méditerrannée.

H. Henninger

team McTAO, Inria Sophia Antipolis Méditerrannée,

2004 rte des lucioles, B.P. 92, 06902 Sophia Antipolis cedex, France.

E-mail: helen-clare.henninger@inria.fr

J. Němcová

Department of Mathematics, Institute of Chemical Technology,

Technická 5, 16628 Prague 6, Czech Republic.

E-mail: jana.nemcova@vscht.cz

J.-B. Pomet

team McTAO, Inria Sophia Antipolis Méditerrannée,

2004 rte des lucioles, B.P. 92, 06902 Sophia Antipolis cedex, France.

E-mail: jean-baptiste.pomet@inria.fr 
spacecraft on this ellipse; $(q, \dot{q})$ can be expressed in terms of $(x, l)$ and vice versa. Restricting to the coplanar case, where $q$ and $\dot{q}$ have dimension 2 and $x$ has dimension 3 , the system can be written as

$$
\dot{x}=\sum_{i=1,2} u_{i} F_{i}(x, l), \quad \dot{l}=\Omega(x, l),
$$

where the control $u=\left(u_{1}, u_{2}\right)$ is the coordinates of the original acceleration $u$ in some frame $F_{1}, F_{2}$, e.g., the tangential/normal frame (the vector fields $F_{1}, F_{2}$ are another basis of the distribution spanned by $\partial / \partial \dot{q}_{1}, \partial / \partial \dot{q}_{2}$ in the original cartesian coordinates). In these coordinates, the free motion is $\dot{x}=0, i=\Omega(x, l)$; there may be a control term in $i$ too but we neglect it for clarity.

The energy minimization problem is the one of minimizing a quadratic criterion $\int\|u\|^{2} \mathrm{~d} t$ for fixed initial and final value of $x$, and free $l$; it was analyzed from the averaging point of view in a series of articles [8,9], [12,11], [4]. The Pontryagin maximum principle yields (for any type of cost: energy, final time or others) an Hamiltonian on the cotangent bundle of the state space with the property that a minimizing trajectory must be the projection of an integral curve of the Hamiltonian vector field. For energy minimization, this Hamiltonian is

$$
H(x, p, l)=\frac{1}{2}\left(H_{1}(x, p, l)^{2}+H_{2}(x, p, l)^{2}\right)
$$

where $H_{i}(x, p, l)=\left\langle p, F_{i}(x, l)\right\rangle$ are the Hamiltonian lifts of the vector fields $F_{i}$ and $p$ is the vector of costate variables of the same dimension as the state vector.

As the bound $\varepsilon$ tends to zero, the time needed to reach a given orbit tends to infinity. During this very long time, the variable $x$ move slowly because the control is small while variables like $l$ move fast thanks to the term $\Omega$; this yields ill conditioned integration if numeric methods are used. It may be shown that there is an average Hamiltonian

$$
H(x, p)=\frac{1}{2 \pi} \int_{0}^{2 \pi} \varpi(x, l) H(x, p, l) \mathrm{d} l,
$$

with $\varpi$ some weight function to be determined, that eliminates the fast variable $l$ and whose Hamiltonian flow gives a remarkably good approximation of the movement of $x$ in the original system if $\varepsilon$ is indeed small. It sometimes leads to explicit formulas, and is anyway much better conditioned numerically because the fast variable has been eliminated.

We shall recall briefly these facts but are more interested in studying qualitatively this new Hamiltonian. We refer the reader to [1, §52] (although no control is considered there) for details on this approximation and its validity. It turns out that it is quadratic definite positive with respect to $p$ and hence derives from a Riemannian metric on $X$; furthermore, the coefficients of this metric can be explicitly computed. In the coplanar case the geodesic flow is Liouville integrable and the metric associated to a subproblem related to transfer from an arbitrary orbit (in $X$ ) to a circular one is even flat: in suitable coordinates the minimizing solutions are straight lines [4]. Moreover this result is still true if the thrust is oriented only in the tangential direction [5].

The same averaging technique can be applied in the minimum time case. The non averaged Hamiltonian reads $\sqrt{H_{1}^{2}(x, p, l)+H_{2}(x, p, l)^{2}}$ and again an averaged Hamiltonian may be constructed:

$$
H(x, p)=\frac{1}{2 \pi} \int_{0}^{2 \pi} \varpi(x, l) \sqrt{H_{1}^{2}(x, p, l)+H_{2}(x, p, l)^{2}} \mathrm{~d} l .
$$

Like in the energy case, this Hamiltonian derives from a metric on $X$, i.e. the data of a norm on each tangent space to $X$; however, unlike in the energy case and as observed in the article [3], these norms are not associated with inner products on these tangent spaces — this defines a Finsler metric [2], not necessarily Riemannian- and are not everywhere smooth. Technical problems involved in going from Riemannian to non smooth Finsler geometry make the computations of time minimal transfer towards circular orbits a complicated problem.

The objective of this article is to make a preliminary qualitative description of the time minimum transfers and to compare them with the energy minimum ones: section 2 recalls the equations and the computation of the average Hamiltonians; section 3 recalls the results from [4,5] on the minimum energy problem; section 4 provides a new analysis of the minimum time problem, for transfers to circular orbits, and in particular proves that the elliptic domain is geodesically convex in this case; section 5 explains why that proof fails in the minimum energy problem, which is consistent with the non-convexity mentioned in [4]. 


\section{Preliminaries}

2.1 Hamiltonian formalism, Pontryagin maximum principle

The goal of this paper is to study some Hamiltonian systems associated to optimal control problems. For the sake of self containedness, let us sketch the relation to the optimal control problems.

Consider the smooth control system $\dot{x}=f(x, u, t)$ for $x \in X$, an $n$-dimensional manifold, $t \in \mathbb{R}$ and $u \in B \subset \mathbb{R}^{\ell}$.

An optimal control problem on $X$ associated with the control system $\dot{x}=f(x, u, t)$ is, for instance, the problem of finding relative to the given points $x_{0}, x_{T}$ the trajectory $x(\cdot)$ and control $u(\cdot)$, and possibly the final time $T$ if it is not specified, such that

$$
\begin{aligned}
\dot{x} & =f(x, u, t), \quad x \in X, \quad\left(u_{1}, u_{2}, \ldots, u_{\ell}\right) \in B \subset \mathbb{R}^{\ell} \\
x(0) & =x_{0}, \quad x(T)=x_{T} \\
\mathcal{J} & =\int_{0}^{T} \mathcal{L}(x(t), u(t)) d t \rightarrow \text { Min. }
\end{aligned}
$$

We call "minimum time" the problem where $\mathcal{L}(x, u)=1$ and $T$ is free, and "minimum energy" the one where $T$ is fixed and $\mathcal{L}(x, u)=\|u\|^{2}$.

The Hamiltonian of the optimal control problem (2) is the function

$$
\mathcal{H}\left(x, p, u, p_{0}, t\right)=p_{0} \mathcal{L}(x, u)+\langle p, f(x, u, t)\rangle
$$

where $p$ is a vector of costate variables (the adjoint vector) of the same dimension as the state variables $x(t)$, and $p_{0}$ is either 0 or -1 . The Pontryagin maximum principle [15] (see also [6, Chap. 6] for applications to the problems we consider here) is a powerful necessary condition for optimality, that states the following: if $(x(\cdot), u(\cdot))$ is an optimal trajectory-control pair of the above optimal control problem on a time interval $[0, T]$, then it can be lifted to a parameterized curve $t \mapsto(x(t), p(t))$ on the cotangent bundle $T^{\star} X$ ( $p$ is the adjoint vector, or the vector of costate variables) that satisfies, for almost all time and either for $p_{0}=0$ or for $p_{0}=-1$,

$$
\begin{aligned}
\dot{x}(t) & =\frac{\partial \mathcal{H}}{\partial p}\left(x(t), p(t), u(t), p_{0}, t\right)=f(x(t), u(t), t) \\
\dot{p}(t) & =-\frac{\partial \mathcal{H}}{\partial p}\left(x(t), p(t), u(t), p_{0}, t\right)
\end{aligned}
$$

and, for almost all $t, \mathcal{H}\left(x(t), p(t), u(t), p_{0}, t\right)$ is the maximum of $\mathcal{H}\left(x(t), p(t), u, p_{0}, t\right)$ with respect to $u \in B$. The solutions where $p_{0}=0$ are called abnormal. Let us assume $p_{0}=-1$.

In the problems we consider here, we are in the nice situation where for all $(x, p, t)$, or almost all $(x, p, t)$, there is a unique $u^{\star}(x, p, t)$ such that

$$
H(x, p, t)=\mathcal{H}\left(x, p, u^{\star}(x, p, t),-1, t\right)=\max _{u \in B} \mathcal{H}(x, p, u,-1, t)
$$

(the second equality is a property of $u^{\star}(x, p, t)$; the first equality is the definition of $H$ from $\mathcal{H}$ and $\left.u^{\star}\right)$. In that case, one may sum up the above in the following way: if $(x(\cdot), u(\cdot))$ is an optimal trajectory, then $x($.$) may be$ lifted to a solution of the Hamiltonian vector field associated to $H$ on $T^{\star} X$ :

$$
\dot{x}=\frac{\partial H}{\partial p}(x, p, t), \quad \dot{p}=-\frac{\partial H}{\partial x}(x, p, t) .
$$

The situation is even nicer if $u^{\star}$ is a smooth function of $x, p, t$; if not, one must be careful about existence and uniqueness of solutions of solutions to this differential equation.

We kept the above time-varying system because we will encounter time-periodic Hamiltonians that we average with respect to time, or with respect to a variable that we may view as a new time. 


\subsection{Coordinates}

First of all, we recall the equations describing the planar controlled Kepler problem in the elliptic case (mechanical energy $K$ is negative).

If we chose as coordinates $\left(n, e_{x}, e_{y}, l\right)$ where $n$ is the mean movement $\left(n=\sqrt{1 / a^{3}}=(-2 K)^{3 / 2} ; a\right.$ is the semi-major axis), $\left(e_{x}, e_{y}\right)$ are the coordinates of the eccentricity vector in a fixed frame and $l$ is the "longitude", or the polar angle with respect to a fixed direction, then the elliptic domain is given by $\left\{n>0, e_{x}^{2}+e_{y}^{2}<1\right\}$. The control system is described by the Gauss equations, where $u_{t}, u_{n}$ are the coordinates of the control in the tangential-normal frame:

$$
\begin{gathered}
\dot{n}=-3 n^{2 / 3} \frac{\sqrt{1+2\left(e_{x} \cos l+e_{y} \sin l\right)+e_{x}^{2}+e_{y}^{2}}}{\sqrt{1-e_{x}^{2}-e_{y}^{2}}} u_{t} \\
\dot{e}_{x}=n^{-1 / 3} \frac{\sqrt{1-e_{x}^{2}-e_{y}^{2}}}{\sqrt{1+2\left(e_{x} \cos l+e_{y} \sin l\right)+e_{x}^{2}+e_{y}^{2}}} \\
\times\left[2\left(\cos l+e_{x}\right) u_{t}-\frac{\sin l+2 e_{y}+2 e_{x} e_{y} \cos l-\left(e_{x}^{2}-e_{y}^{2}\right) \sin l}{\sqrt{1-e_{x}^{2}-e_{y}^{2}}} u_{n}\right] \\
\dot{e}_{y}=n^{-1 / 3} \frac{\sqrt{1-e_{x}^{2}-e_{y}^{2}}}{\sqrt{1+2\left(e_{x} \cos l+e_{y} \sin l\right)+e_{x}^{2}+e_{y}^{2}}} \\
\times\left[2\left(\sin l+e_{y}\right) u_{t}-\frac{\cos l+2 e_{x}+\left(e_{x}^{2}-e_{y}^{2}\right) \cos l+2 e_{x} e_{y} \sin l}{\sqrt{1-e_{x}^{2}-e_{y}^{2}}} u_{n}\right] \\
i=n \frac{\left(1+e_{x} \cos l+e_{y} \sin l\right)^{2}}{\left(1-e^{2}\right)^{3 / 2}} .
\end{gathered}
$$

Instead of $e_{x}, e_{y}$, it will be more convenient to use the eccentricity $e$ and the argument of the pericenter $\omega$ (not defined if $e=0$ ), defined by

$$
e_{x}=e \cos \omega, e_{y}=e \sin \omega
$$

The equations become:

$$
\begin{aligned}
& \dot{n}=-\frac{3 n^{2 / 3}}{\sqrt{1-e^{2}}}\left[\sqrt{1+2 e \cos v+e^{2}} u_{t}\right] \\
& \dot{e}=\frac{\sqrt{1-e^{2}}}{\sqrt[3]{n}} \frac{1}{\sqrt{1+2 e \cos v+e^{2}}}\left[2(e+\cos v) u_{t}-\sin v \frac{1-e^{2}}{1+e \cos v} u_{n}\right] \\
& \dot{\omega}=\frac{\sqrt{1-e^{2}}}{e \sqrt[3]{n}} \frac{1}{\sqrt{1+2 e \cos v+e^{2}}}\left[2 \sin v u_{t}+\frac{2 e+\cos v+e^{2} \cos v}{1+e \cos v} u_{n}\right] \\
& \dot{l}=n \frac{(1+e \cos v)^{2}}{\left(1-e^{2}\right)^{3 / 2}} .
\end{aligned}
$$

The angle $v$ is the true anomaly

$$
v=l-\omega
$$

In these coordinates, the elliptic domain is

$$
X=\left\{x=(n, e, \omega), n>0,0 \leq e<1, \omega \in S^{1}\right\} .
$$

Remark 1 (Transfer towards a circular orbit) In the transfer "towards a circular orbit" (or merely if we do not take into account the direction of the semi-major axis during the transfer), we may use these coordinates although they are singular at $e=0$, because the variable $\omega$ may simply be ignored; this is possible because it is a cyclic variable, i.e. it does not influence the evolution of the other variables $(n, e, v)$. In the variables $(n, e)$, the elliptic domain is:

$$
\mathcal{X}=\{(n, e), 0<n<+\infty,-1<e<1\} .
$$

The fact that negative values of $e$ are allowed comes from identifying $(-e, \omega)$ with $(e, \omega+\pi)$, or, equivalently, considering that $\left(e_{x}, e_{y}\right)$ (see (6) ) lies on a line of fixed arbitrary direction instead of a half-line. This line may for instance be $\left\{e_{y}=0\right\}$, and $\mathcal{X}$ is then identified with $\left\{\left(n, e_{x}, e_{y}\right), n>0,-1<e<1, e=e_{x}, e_{y}=0\right\}$. 
Equations (7a)-(7d) read:

$$
\dot{x}=\sum_{1 \leq i \leq 2} u_{i} F_{i}(x, l), \quad i=\Omega(x, l)
$$

where $u_{1}, u_{2}$ stand for $u_{n}, u_{t}, x=(n, e, \omega)$, the vectors $F_{1}, F_{2}$ are readily obtained from (7a)-(7c), and

$$
\Omega(x, l)=n \frac{(1+e \cos (l-\omega))^{2}}{\left(1-e^{2}\right)^{3 / 2}} .
$$

One way to introduce averaging is to use the so-called "mean eccentric anomaly". The eccentric anomaly is $E$, related to $e$ and $v$ by

$$
\tan \frac{v}{2}=\sqrt{\frac{1+e}{1-e}} \tan \frac{E}{2}
$$

and the mean eccentric anomaly is $E-e \sin E$; the Kepler equation (third Kepler law) implies that, when the control is zero,

$$
E-e \sin E=n t,
$$

$t=0$ being the time at the pericenter. Introducing (see for instance [6, sec. 3.6.3])

$$
x_{0}=(E-e \sin E) / n,
$$

one has $\dot{x}_{0}=1$ if $u=0$, i.e. the variable $x_{0}$ behaves like time modulo an additive constant; this is an implementation of the flow-box theorem. In the coordinates $\left(x, x_{0}\right)$, the system becomes

$$
\dot{x}=\sum_{i=1,2} u_{i} \widehat{F}_{i}\left(x, x_{0}\right), \quad \dot{x}_{0}=1+\sum_{i=1,2} u_{i} G_{i}\left(x, x_{0}\right) .
$$

Due to the implicit relation between $E$ and $x_{0}$, the practical derivation of such equations is complicated, but they will be useful in formally identifying averaging with respect to $l \in[0,2 \pi]$ and averaging with respect to $t \in[0,2 \pi / n]$.

We define the Hamiltonian lifts $(i=1,2)$ :

$$
H_{i}(x, p, l)=\left\langle p, F_{i}(x, p, l)\right\rangle, \quad \widehat{H}_{i}\left(x, p, x_{0}\right)=\left\langle p, \widehat{F}_{i}\left(x, p, x_{0}\right)\right\rangle .
$$

\subsection{Averaging}

Using the previous equations and rescaling the control with $u=\varepsilon v$ to introduce the small parameter, the trajectories parameterized by $x_{0}$ are solutions of

$$
\frac{d x}{d x_{0}}=\frac{\varepsilon \sum_{i=1,2} v_{i} \widehat{F}_{i}\left(x, x_{0}\right)}{1+\varepsilon \sum_{i=1,2} v_{i} G_{i}\left(x, x_{0}\right)}
$$

which is approximated for small $\varepsilon$ by

$$
\frac{d x}{d x_{0}}=\varepsilon \sum_{i=1,2} v_{i} \widehat{F}_{i}\left(x, x_{0}\right)
$$

For this system, we consider the following minimization problems:

- Energy : $\min _{v} \varepsilon^{2} \int_{0}^{x_{0}} \sum_{i=1,2} v_{i}^{2} \mathrm{~d} t$

- Time : $\min _{v} x_{0},\|v\| \leq 1$.

Applying the Pontryagin maximum principle leads to the following respective Hamiltonians (normal case in the energy minimization problem),

$$
H_{\mathrm{e}}\left(x, p, x_{0}\right)=\sum_{i=1,2} \widehat{H}_{i}\left(x, p, x_{0}\right)^{2}, \quad H_{\mathrm{t}}\left(x, p, x_{0}\right)=\sqrt{\sum_{i=1,2} \widehat{H}_{i}\left(x, p, x_{0}\right)^{2}},
$$

where the lifts $\widehat{H}_{i}$, defined by (14), are periodic with respect to $x_{0}$ with period $2 \pi / n$. 
Remark 2 (Tangential thrust) If the normal component $u_{n}$ is forced to be zero, there is a single term in the sums in (15), and these equations become $H_{\mathrm{e}}=\widehat{H}_{1}^{2}, \quad H_{\mathrm{t}}=\left|\widehat{H}_{1}\right|$. The considerations in the present section are valid both in the full control case and in the "tangential thrust" case.

The respective averaged Hamiltonians are

$$
\begin{aligned}
& H_{\mathrm{e}}(x, p)=\frac{n}{2 \pi} \int_{0}^{2 \pi / n} H_{\mathrm{e}}\left(x, p, x_{0}\right) d x_{0} \\
& H_{\mathrm{t}}(x, p)=\frac{n}{2 \pi} \int_{0}^{2 \pi / n} H_{\mathrm{t}}\left(x, p, x_{0}\right) d x_{0} .
\end{aligned}
$$

(for ease of notation we use $H_{\mathrm{e}}, H_{\mathrm{t}}$ to represent both the Hamiltonians and the averaged Hamiltonians, although the inputs into these functions are different). These may be re-computed in terms of $H_{1}, H_{2}$. Unlike when $\widehat{H}_{1}, \widehat{H}_{2}$ are used in the computation, using the Hamiltonian lifts $H_{1}, H_{2}$ allows for an explicit expression of the averaged Hamiltonians $H_{\mathrm{e}}(x, p), H_{\mathrm{t}}(x, p)$. Making the change of variables $x_{0}=\Xi(e, \omega, l)$-with $\Xi$ deduced from $x_{0}=$ $(E-e \sin E) / n,(13)$ and $(8)$ - in the integral, and using the facts that $\partial \Xi / \partial l=1 / \Omega(x, l)$ and

$$
\widehat{H}_{\mathrm{e}}(x, p, \Xi(e, \omega, l))=H_{\mathrm{e}}(x, p, l), \widehat{H}_{\mathrm{t}}(x, p, \Xi(e, \omega, l))=H_{\mathrm{t}}(x, p, l),
$$

then, using (12),

$$
\begin{gathered}
H_{\mathrm{e}}(x, p)=\frac{\left(1-e^{2}\right)^{3 / 2}}{2 \pi} \int_{0}^{2 \pi}\left(\sum_{i=1,2} H_{i}(x, p, l)^{2}\right) \frac{\mathrm{d} l}{(1+e \cos (l-\omega))^{2}} \\
H_{\mathrm{t}}(x, p)=\frac{\left(1-e^{2}\right)^{3 / 2}}{2 \pi} \int_{0}^{2 \pi} \sqrt{\sum_{i} H_{i}(x, p, l)^{2}} \frac{\mathrm{d} l}{(1+e \cos (l-\omega))^{2}} .
\end{gathered}
$$

Remark 3 In the original system, the control is "small" (parameter $\varepsilon$ ). The average system that we study in the next sections can be seen as a limit as $\varepsilon \rightarrow 0$.

The smaller $\varepsilon$ is, the better the average system approximates the real system, but neither the results of this paper not any analysis or simulation in the next sections depend on the size of $\varepsilon$, that is on the magnitude of the thrust.

Singularities. Let us explain how the non smoothness is a result of the averaging of singularities of a control system. Consider the time minimal control problem for a generic smooth system of the form

$$
\dot{x}=F_{0}(x)+\sum_{i=1, m} u_{i} F_{i}(x), \quad\|u\| \leqq 1 .
$$

Moreover assume for simplicity that the control distribution $D=\operatorname{span}\left\{F_{1}, \ldots, F_{m}\right\}$ is involutive. From the maximum principle in this case, the extremal control is defined by $u_{i}=\frac{H_{i}(x, p)}{\sqrt{\sum_{i} H_{i}^{2}(x, p)}}$ where $H_{i}(x, p)$ are the Hamiltonian lifts of $F_{i}(x)$. More complicated extremals are related to the switching surface $\Sigma: H_{i}=0$. Observe that in the single-input case the control is given by $u_{1}=\operatorname{sign} H_{1}(x, p)$ and meeting the surface $\Sigma$ transversally corresponds to a regular switching. This can be generalized to the multi-input case. More complicated singularities can occur in the non transversal case, for instance in relation with singular trajectories of the system (contained by definition in the surface $\Sigma$ ) [7].

\section{The analysis of the averaged systems for minimum energy}

First of all we recall the results from the energy case [4] . The energy minimization problem is expressed as

$$
\int_{0}^{l_{f}}\left(u_{1}^{2}(t)+u_{2}^{2}(t)\right) d t \rightarrow \text { Min }
$$

where we fix the final cumulated longitude $l_{f}$ (this is slightly different from fixing the transfer time). 
3.1 The coplanar energy case

In this case the averaged system can be computed explicitly by quadrature, and we have the following proposition.

Proposition 1 In the coordinates $(n, e, \omega)$ the averaged Hamiltonian (up to a positive scalar) is given by

$$
H_{\mathrm{e}}=\frac{1}{n^{5 / 3}}\left[18 n^{2} p_{n}^{2}+5\left(1-e^{2}\right) p_{e}^{2}+\frac{5-4 e^{2}}{e^{2}} p_{\omega}^{2}\right]
$$

where the singularity $e=0$ corresponds to circular orbits. In particular $(n, e, \omega)$ are orthogonal coordinates for the Riemannian metric associated to $H$, namely

$$
g=\frac{1}{9 n^{1 / 3}} \mathrm{~d} n^{2}+\frac{2 n^{5 / 3}}{5\left(1-e^{2}\right)} \mathrm{d} e^{2}+\frac{2 n^{5 / 3}}{5-4 e^{2}} \mathrm{~d} \omega^{2} .
$$

Further normalizations are necessary to capture the main properties of the averaged orbital transfer.

Proposition 2 In the elliptic domain we set

$$
r=\frac{2}{5} n^{5 / 6}, \varphi=\arcsin e
$$

and the metric is isometric to

$$
g=\mathrm{d} r^{2}+\frac{r^{2}}{c^{2}}\left(\mathrm{~d} \varphi^{2}+G(\varphi) \mathrm{d} \omega^{2}\right)
$$

where $c=\sqrt{2 / 5}$ and $G(\varphi)=\frac{5 \sin ^{2} \varphi}{1+4 \cos ^{2} \varphi}$.

\subsection{Transfer towards circular orbits}

As noticed in Remark 1, for such transfers we may ignore the cyclic variable $\omega$ and allow negative $e$. In this case, the elliptic domain is the $\mathcal{X}$ given by $(10)$. The metric above then reduces to

$$
g=\mathrm{d} r^{2}+r^{2} d \psi^{2}, \text { with } \psi=\varphi / c
$$

defined on the domain $\left\{(r, \psi), 0<r<+\infty,-\frac{\pi}{2 c}<\psi<\frac{\pi}{2 c}\right\}$; it is a polar metric isometric to the flat metric $d x^{2}+d z^{2}$ if we set $x=r \sin \psi$ and $z=r \cos \psi$. Flatness in the original coordinates can be checked by computing the Gauss curvature. We deduce the following theorem:

Theorem 1 The geodesics of the averaged coplanar transfer towards circular orbits are straight lines in the domain $\mathcal{X}$ (see (10)) in suitable coordinates, namely

$$
x=\frac{2^{3 / 2}}{5} n^{5 / 6} \sin \left(\frac{1}{c} \arcsin e\right), z=\frac{2^{3 / 2}}{5} n^{5 / 6} \cos \left(\frac{1}{c} \arcsin e\right)
$$

with $c=\sqrt{2 / 5}$. Since $c<1$, the domain is not (geodesically) convex and the metric is not complete.

Remark 4 (Tangential thrust) The properties of theorem 1 are still true when the thrust is only in the tangential direction except that the metric has a singularity at $e=1$. The formula is

$$
g=\frac{1}{9 n^{1 / 3}} \mathrm{~d} n^{2}+\frac{\left(1+\sqrt{1-e^{2}}\right) n^{5 / 3}}{4\left(1-e^{2}\right)}\left[\frac{1}{\sqrt{1-e^{2}}} \mathrm{~d} e^{2}+e^{2} \mathrm{~d} \omega^{2}\right] .
$$

We may slightly twist the previous coordinates using $e=\sin \varphi \sqrt{1+\cos ^{2} \varphi}$ to get the normal form $\mathrm{d} r^{2}+$ $\left(r^{2} / c_{t}\right)\left(\mathrm{d} \varphi^{2}+G_{t}(\varphi) \mathrm{d} \omega^{2}\right), c_{t}=c^{2}=2 / 5, G_{t}(\varphi)=\sin ^{2} \varphi\left(\frac{1-(1 / 2) \sin ^{2} \varphi}{1-\sin ^{2} \varphi}\right)^{2}$. 


\section{The analysis of the averaged systems for minimum time}

\subsection{The Hamiltonian}

We compute $H_{\mathrm{t}}$ according to (19). The functions $H_{i}, i=1,2$ depend on $n, e, \omega, p_{n}, p_{e}, p_{\omega}, l$. Since we only consider transfer towards a circular orbit, we set $p_{\omega}=0$ and define $h_{1}, h_{2}$ by

$$
h_{i}\left(n, e, p_{n}, p_{e}, v\right)=H_{i}\left(n, e, \omega, p_{n}, p_{e}, 0, \omega+v\right) .
$$

The right-hand side does not depend on the cyclic variable $\omega$, see Remark 1. From here on we will use the subscripts 1 and 2 to denote respectively the tangential and normal directions, rather than $t$ and $n$, for ease of notation. From (7), we get

$$
\begin{aligned}
& h_{1}=n^{-1 / 3}\left(-3 n p_{n} \frac{\sqrt{1+2 e \cos v+e^{2}}}{\sqrt{1-e^{2}}}+2 p_{e} \frac{(e+\cos v) \sqrt{1-e^{2}}}{\sqrt{1+2 e \cos v+e^{2}}}\right) \\
& h_{2}=-n^{-1 / 3} p_{e} \frac{\sin v\left(1-e^{2}\right)^{3 / 2}}{(1+e \cos v) \sqrt{1+2 e \cos v+e^{2}}}
\end{aligned}
$$

Note that $\omega$ does not vary in the integral; the integrand has period $2 \pi$ with respect to either $l$ or $v$. This allows us to make the change of variable $l=\omega+v$ in the integral in (19). In the full control case (both tangential and normal control), the sum in (19) contains two terms, and we obtain

$$
H_{\mathrm{t}}\left(n, e, p_{n}, p_{e}\right)=\frac{\left(1-e^{2}\right)^{3 / 2}}{2 \pi} \int_{0}^{2 \pi} \sqrt{\sum_{i=1}^{2} h_{i}\left(n, e, p_{n}, p_{e}, v\right)^{2}} \frac{\mathrm{d} v}{(1+e \cos v)^{2}},
$$

In the tangential thrust case it only contains $h_{1}$ - see remark 2 - and we get (the superscript 1 in $H_{\mathrm{t}}^{1}$ denotes single input):

$$
H_{\mathrm{t}}^{1}\left(n, e, p_{n}, p_{e}\right)=\frac{\left(1-e^{2}\right)^{3 / 2}}{2 \pi} \int_{0}^{2 \pi}\left|h_{1}\left(n, e, p_{n}, p_{e}, v\right)\right| \frac{\mathrm{d} v}{(1+e \cos v)^{2}} .
$$

In order to highlight some properties of these Hamiltonians, we perform a canonical change of coordinates $\left(n, e, p_{n}, p_{e}\right) \mapsto\left(\lambda, \varphi, p_{\lambda}, p_{\varphi}\right)$ :

$$
n=e^{3 \lambda}, e=\sin \varphi, p_{n}=\frac{p_{\lambda}}{3 n}, p_{e}=\frac{p_{\varphi}}{\cos \varphi}
$$

followed by taking $(\rho, \psi)$ as polar coordinated for the adjoint vector $\left(p_{\lambda}, p_{\varphi}\right)$; we shall never use again the notations $\lambda, p_{\lambda}, p_{\varphi}$ and directly write the change as

$$
3 n p_{n}=\rho \cos \psi, \quad \sqrt{1-e^{2}} p_{e}=\rho \sin \psi, \quad e=\sin \varphi,-\frac{\pi}{2}<\varphi<\frac{\pi}{2} .
$$

Equations (23) and (24) then yield

$$
\begin{aligned}
& H_{\mathrm{t}}\left(n, \sin \varphi, \frac{\rho \cos \psi}{3 n}, \frac{\rho \sin \psi}{\cos \varphi}\right)=\rho n^{-1 / 3} L(\psi, \varphi) \\
& H_{\mathrm{t}}^{1}\left(n, \sin \varphi, \frac{\rho \cos \psi}{3 n}, \frac{\rho \sin \psi}{\cos \varphi}\right)=\rho n^{-1 / 3} M(\psi, \varphi)
\end{aligned}
$$

with $L$ and $M$ some functions $\mathcal{C} \rightarrow \mathbb{R}$, where $\mathcal{C}$ is the cylinder

$$
\mathcal{C}=\left\{(\psi, \varphi), \psi \in(\mathbb{R} / 2 \pi \mathbb{Z}), \varphi \in \mathbb{R},-\frac{\pi}{2}<\varphi<\frac{\pi}{2}\right\}=\mathbb{R} / 2 \pi \mathbb{Z} \times\left(-\frac{\pi}{2}, \frac{\pi}{2}\right) .
$$

The expressions of $L$ and $M$ are, taking the eccentric anomaly $E$ as the variable of integration instead of $v$ (see (13); in particular, $\left.\mathrm{d} v /(1+e \cos v)=\mathrm{d} E / \sqrt{1-e^{2}}\right)$ and restricting the interval of integration from $[0,2 \pi]$ to $[0, \pi]$ because the integrand depends on $\cos E$ only:

$$
\begin{aligned}
& L(\psi, \varphi)=\frac{1}{\pi} \int_{0}^{\pi} \sqrt{\widetilde{I}(\psi, \varphi, E)} \mathrm{d} E, \\
& \widetilde{I}(\psi, \varphi, E)=\alpha^{1,1}(\varphi, \cos E) \cos ^{2} \psi+2 \alpha^{1,2}(\varphi, \cos E) \cos \psi \sin \psi+\alpha^{2,2}(\varphi, \cos E) \sin ^{2} \psi, \\
& \alpha^{1,1}=1-\sin ^{2} \varphi \cos ^{2} E, \quad \alpha^{1,2}=-2 \cos \varphi(1-\sin \varphi \cos E) \cos E, \\
& \alpha^{2,2}=(1-\sin \varphi \cos E)\left(1-3 \sin \varphi \cos E+3 \cos ^{2} E-\sin \varphi \cos ^{3} E\right)
\end{aligned}
$$


and

$$
\begin{aligned}
& M(\psi, \varphi)=\frac{1}{\pi} \int_{0}^{\pi}|\widetilde{J}(\psi, \varphi, E)| \mathrm{d} E \\
& \widetilde{J}(\psi, \varphi, E)=\sqrt{\frac{1-\sin \varphi \cos E}{1+\sin \varphi \cos E}}((2 \cos \varphi \sin \psi-\sin \varphi \cos \psi) \cos E-\cos \psi) .
\end{aligned}
$$

In the sequel we take advantage of the double homogeneity with respect to $\rho$ and $n$ displayed in (26) and (27).

4.2 Singularities of the Hamiltonian in the single-input and two-input cases

According to 26) and 27], the Hamiltonians $H_{\mathrm{t}}$ and $H_{\mathrm{t}}^{1}$, have the same degree of smoothness as, respectively the maps $L$ and $M$.

Proposition 3 The maps $L: \mathcal{C} \rightarrow \mathbb{R}$ and $M: \mathcal{C} \rightarrow \mathbb{R}$ are real analytic away from

$$
\mathcal{S}=\left\{(\psi, \varphi), \tan \psi=\frac{1+\sin \varphi}{2 \cos \varphi}\right\} \cup\left\{(\psi, \varphi), \tan \psi=\frac{-1+\sin \varphi}{2 \cos \varphi}\right\} .
$$

They are both continuously differentiable on $\mathcal{C}$, but their differentials are not locally Lipschitz-continuous on the set $\mathcal{S}$; we have the following moduli of continuity of the differentials: in a neighborhood of a point $\xi=(\psi, \varphi) \in \mathcal{S}$, in some corrdinates and for a "small" $\delta$,

$$
\begin{aligned}
\|\mathrm{d} L(\xi+\delta)-\mathrm{d} L(\xi)\| & \leq k\|\delta\| \ln (1 /\|\delta\|) \\
\|\mathrm{d} M(\xi+\delta)-\mathrm{d} M(\xi)\| & \leq k\|\delta\|^{1 / 2}
\end{aligned}
$$

Proof The set $\mathcal{S}$ is the set of points $(\psi, \varphi)$ such that $\widetilde{I}(\psi, \varphi, E)$ vanishes for some value of $E$; hence the integrand in 29] is real analytic on $\mathcal{C} \backslash \mathcal{S}$ and so is $L$. The degree of regularity [35] for $L$ at points in $\mathcal{S}$ is given in [3].

Let us now treat $M$. It turns out that $\mathcal{S}$ is also the border between the region

$$
\mathcal{R}_{1}=\left\{(\psi, \varphi) \in \mathcal{C}: \frac{-1+\sin \varphi}{2 \cos \varphi}<\tan \psi<\frac{1+\sin \varphi}{2 \cos \varphi}\right\}
$$

where the sign of $\widetilde{J}(\psi, \varphi, E)$ does not depend on $E$ and the region

$$
\mathcal{R}_{2}=\left\{(\psi, \varphi) \in \mathcal{C}: \tan \psi<\frac{-1+\sin \varphi}{2 \cos \varphi} \text { or } \frac{1+\sin \varphi}{2 \cos \varphi}<\tan \psi\right\}
$$

where $\widetilde{J}(\psi, \varphi, E)$ vanishes for two distinct values of the angle $E$ where it changes sign; these two values are given by $\cos E=R(\psi, \varphi)$ with

$$
R(\psi, \varphi)=\frac{\cos \psi}{P(\psi, \varphi)}, \quad P(\psi, \varphi)=2 \cos \varphi \sin \psi-\sin \varphi \cos \psi
$$

(note that (37), (38) amount to $\mathcal{R}_{1}=\{|R(\psi, \varphi)|>1\}, \mathcal{R}_{2}=\{|R(\psi, \varphi)|<1\}$ and $\mathcal{S}$ is the locus where $R= \pm 1$ ). Hence (32) yields

$$
M(\psi, \varphi)= \begin{cases}\frac{-\operatorname{sign} \cos \psi}{\pi} \int_{0}^{\pi} \widetilde{J}(\psi, \varphi, E) \mathrm{d} E & \text { on } \mathcal{R}_{1}, \\ \frac{\operatorname{sign} P(\psi, \varphi)}{\pi}\left(\int_{0}^{\arccos R(\psi, \varphi)} \widetilde{J}(\psi, \varphi, E) \mathrm{d} E-\int_{\arccos R(\psi, \varphi)}^{\pi} \widetilde{J}(\psi, \varphi, E) \mathrm{d} E\right) & \text { on } \mathcal{R}_{2} .\end{cases}
$$

It is therefore clear that $M$ is real analytic on $\mathcal{C} \backslash \mathcal{S}=\mathcal{R}_{1} \cup \mathcal{R}_{2}$. The singularity of $M$ on $\mathcal{S}$ is not of the type treated in [3], but it is clear above that the restriction of $M$ to $\mathcal{R}_{1}$ has a real analytic continuation through $\mathcal{S}$ while its restriction to $\mathcal{R}_{2}$, on the contrary, behaves like a square root in a neighborhood of $\mathcal{S}$, whence (36).

The properties of the differential of the Hamiltonian are important because it is the right-hand side of the Hamiltonian equation. Studying these singularities more precisely is an interesting program that is not yet carried out. 
Let us now study the solutions of the Hamiltonian equation associated with the minimum time problem in the full control or single control (tangential thrust) cases, namely:

$$
\dot{n}=\frac{\partial H_{\mathrm{t}}}{\partial p_{n}}, \dot{e}=\frac{\partial H_{\mathrm{t}}}{\partial p_{e}}, \dot{p}_{n}=-\frac{\partial H_{\mathrm{t}}}{\partial n}, \dot{p}_{e}=-\frac{\partial H_{\mathrm{t}}}{\partial e}
$$

and

$$
\dot{n}=\frac{\partial H_{\mathrm{t}}^{1}}{\partial p_{n}}, \quad \dot{e}=\frac{\partial H_{\mathrm{t}}^{1}}{\partial p_{e}}, \quad \dot{p}_{n}=\frac{\partial H_{\mathrm{t}}^{1}}{\partial n}, \quad \dot{p}_{e}=\frac{\partial H_{\mathrm{t}}^{1}}{\partial e} .
$$

with $H_{\mathrm{t}}$ given by (23) and $H_{\mathrm{t}}^{1}$ by 24 ).

Specifically, we establish geodesic convexity of the elliptic domain $\mathcal{X}$ (see $(10)$ ), i.e. any two points in $\mathcal{X}$ can be joined by a extremal curve. This is contained in the following result:

Theorem 2 (geodesic convexity) For any $\left(n^{0}, e^{0}\right)$ and $\left(n^{1}, e^{1}\right)$ in $\mathcal{X}$, there exist a time $T \geq 0$ and a solution $[0, T] \rightarrow \mathcal{X}, t \mapsto\left(n(t), e(t), p_{n}(t), p_{e}(t)\right)$ of (41) (resp. of (42)) such that $(n(0), e(0))=\left(n^{0}, e^{0}\right)$ and $(n(T), e(T))=\left(n^{1}, e^{1}\right)$.

In order to ease the proof, let us write 41, and (42) in other coordinates.

Proposition 4 In the coordinates $(n, \varphi, \psi, \rho)$ defined by 25, and after a time re-parametrization

$$
\mathrm{d} t=n^{1 / 3} \mathrm{~d} \tau
$$

equation (41) (resp. equation (42)) becomes

$$
\frac{\mathrm{d} \psi}{\mathrm{d} \tau}=a(\psi, \varphi), \quad \frac{\mathrm{d} \varphi}{\mathrm{d} \tau}=b(\psi, \varphi), \quad \frac{\mathrm{d} n}{\mathrm{~d} \tau}=-3 n c(\psi, \varphi)
$$

where $a, b, c$ are given $b \sqrt{1}$.

$$
\begin{aligned}
& a(\psi, \varphi)=-L(\psi, \varphi) \sin \psi-L_{\varphi}(\psi, \varphi) \cos \psi \\
& b(\psi, \varphi)=L(\psi, \varphi) \sin \psi+L_{\psi}(\psi, \varphi) \cos \psi \\
& c(\psi, \varphi)=L(\psi, \varphi) \cos \psi-L_{\psi}(\psi, \varphi) \sin \psi
\end{aligned}
$$

(resp. given by:

$$
\begin{aligned}
& a(\psi, \varphi)=-M(\psi, \varphi) \sin \psi-M_{\varphi}(\psi, \varphi) \cos \psi, \\
& b(\psi, \varphi)=M(\psi, \varphi) \sin \psi+M_{\psi}(\psi, \varphi) \cos \psi, \\
& c(\psi, \varphi)=M(\psi, \varphi) \cos \psi-M_{\psi}(\psi, \varphi) \sin \psi,
\end{aligned}
$$

and the evolution of $\rho$ is given by:

$$
\begin{gathered}
\rho(\tau)=\rho(0)\left(\frac{n(0)}{n(\tau)}\right)^{-1 / 3} \frac{L(\psi(0), \varphi(0))}{L(\psi(\tau), \varphi(\tau))} \\
\left(\text { resp. } \rho(\tau)=\rho(0)\left(\frac{n(0)}{n(\tau)}\right)^{-1 / 3} \frac{M(\psi(0), \varphi(0))}{M(\psi(\tau), \varphi(\tau))}\right) .
\end{gathered}
$$

The "time" $\tau$ is related to the real time $t$ by

$$
\begin{aligned}
t & =\frac{n(\tau)^{1 / 3} \cos \psi(\tau)}{L(\varphi(\tau), \psi(\tau))}-\frac{n(0)^{1 / 3} \cos \psi(0)}{L(\varphi(0), \psi(0))} \\
\text { (resp. } t & \left.=\frac{n(\tau)^{1 / 3} \cos \psi(\tau)}{M(\psi(\tau), \varphi(\tau))}-\frac{n(0)^{1 / 3} \cos \psi(0)}{M(\psi(0), \varphi(0))}\right) .
\end{aligned}
$$

\footnotetext{
1 lower indices stand for partial derivatives
} 
Proof From (25) and (41) (resp. (25) and (42)), one gets

$$
\begin{aligned}
& \dot{\psi}=\frac{1}{\rho}\left(3 n \sin \psi \frac{\partial H}{\partial n}-\cos \varphi \cos \psi \frac{\partial H}{\partial e}\right)-\cos \psi \sin \psi\left(\frac{1}{n} \frac{\partial H}{\partial p_{n}}+\frac{\sin \varphi}{\cos ^{2} \varphi} \frac{\partial H}{\partial p_{e}}\right), \\
& \dot{\varphi}=\frac{1}{\cos \varphi} \frac{\partial H}{\partial p_{e}}, \quad \dot{n}=\frac{\partial H}{\partial p_{n}}
\end{aligned}
$$

where $H$ stands for $H_{\mathrm{t}}$ (resp. for $H_{\mathrm{t}}^{1}$ ). Differentiating (26) (resp. 27p) with respect to $n, \varphi, \rho, \psi$ and solving for $\frac{\partial H_{\mathrm{t}}}{\partial n}, \frac{\partial H_{\mathrm{t}}}{\partial e}, \frac{\partial H_{\mathrm{t}}}{\partial p_{n}}, \frac{\partial H_{\mathrm{t}}}{\partial p_{e}}$ (resp. for $\frac{\partial H_{\mathrm{t}}^{1}}{\partial n}, \frac{\partial H_{\mathrm{t}}^{1}}{\partial e}, \frac{\partial H_{\mathrm{t}}^{1}}{\partial p_{n}}, \frac{\partial H_{\mathrm{t}}^{1}}{\partial p_{e}}$ ), we obtain the latter as linear combinations of $L(\psi, \varphi)$, $L_{\varphi}(\psi, \varphi), L_{\psi}(\psi, \varphi)$ (resp. of $M(\psi, \varphi), M_{\varphi}(\psi, \varphi), M_{\psi}(\psi, \varphi)$ ) with coefficients depending on $n, \varphi, \rho, \psi$; substituting these expressions into (51) gives

$$
\begin{aligned}
\dot{\psi} & =n^{-1 / 3}\left(-L \sin \psi-L_{\varphi} \cos \psi\right), \\
\dot{\varphi} & =n^{-1 / 3}\left(L \sin \psi+L_{\psi} \cos \psi\right), \\
\dot{n} & =-3 n^{2 / 3}\left(L \cos \psi-L_{\psi} \sin \psi\right) \\
\text { (resp. } \quad \dot{\psi} & =n^{-1 / 3}\left(-M \sin \psi-M_{\varphi} \cos \psi\right), \\
\dot{\varphi} & =n^{-1 / 3}\left(M \sin \psi+M_{\psi} \cos \psi\right), \\
\dot{n} & \left.=-3 n^{2 / 3}\left(M \cos \psi-M_{\psi} \sin \psi\right) \quad\right) .
\end{aligned}
$$

With the new time $\tau$ given by (43), one easily deduces (44) and the expressions (45) (resp. (46) of $a, b, c$. Finally, (44) and 45) (resp. (44) and (46) imply $\frac{\mathrm{d}}{\mathrm{d} \tau}\left(\frac{n^{1 / 3} \cos \psi}{L(\psi, \varphi)}\right)=n^{-1 / 3}$ (resp. $\frac{d}{d \tau}\left(\frac{n^{1 / 3} \cos \psi}{M(\psi, \varphi)}\right)=n^{1 / 3}$ ), that implies (49) (resp. (50)) according to (43).

The first two equations in (44) form an autonomous system of equations in the two variables $(\psi, \varphi) \in \mathcal{C}$ that will be the core of our analysis; the third one may be integrated and yields $n(\tau)$ :

$$
n(\tau)=n(0) \exp \left(-3 \int_{0}^{\tau} c(\psi(\sigma), \varphi(\sigma)) \mathrm{d} \sigma\right) .
$$

The variable $\rho$ (the magnitude of the adjoint vector) plays no role in the evolution of the other variables, in particular the state $(n, e)(e=\sin \varphi)$; this is a well-known consequence of the Hamiltonian being homogeneous of degree 1 with respect to the adjoint vector and is anyway obvious from (44).

Let us now gather some properties of the maps $a, b, c$, i.e. of the differential equation (44), that are valid both for $a, b, c$ given by (45) and for $a, b, c$ given by (46); they contain all the information to prove Theorem 2

Proposition 5 The maps $a, b, c$ given by (45) satisfy the following properties with $\bar{\sigma}=0$. The maps a, $b, c$ given by (46) satisfy the same properties with $\bar{\sigma}=\arctan \frac{1}{2}$.

1. Symmetries. For all $(\psi, \varphi)$ in $\mathcal{C}$,

$$
\begin{array}{llrl}
a(\psi+\pi, \varphi) & =-a(\psi, \varphi), & & a(-\psi,-\varphi)=-a(\psi, \varphi), \\
b(\psi+\pi, \varphi)=-b(\psi, \varphi), & & b(-\psi,-\varphi)=-b(\psi, \varphi), \\
c(\psi+\pi, \varphi) & =-c(\psi, \varphi), & & c(-\psi,-\varphi)=c(\psi, \varphi) .
\end{array}
$$

2. Uniqueness of solutions. The following differential equation on $\mathcal{C}$ :

$$
\dot{\psi}=a(\psi, \varphi), \quad \dot{\varphi}=b(\psi, \varphi)
$$

has, for any $\left(\psi^{o}, \varphi^{o}\right) \in \mathcal{C}$, a unique solution $t \mapsto(\psi(t), \varphi(t))$ such that $(\psi(0), \varphi(0))=\left(\psi^{o}, \varphi^{o}\right)$, defined on a maximum open interval of definition $\left(\tau^{-}, \tau^{+}\right)$. In this interval, $\tau^{-}<0<\tau^{+}$where $\tau^{-}$is such that either $\tau^{-}=-\infty$ or $\varphi\left(\tau^{-}\right)= \pm \frac{\pi}{2}$, and $\tau^{+}$is such that either $\tau^{+}=+\infty$ or $\varphi\left(\tau^{+}\right)= \pm \frac{\pi}{2}$. This defines a flow $\Phi$ from an open subset of $\mathcal{C} \times \mathbb{R}$ to $\mathcal{C}$ such that the above unique solution is

$$
t \mapsto \Phi\left(\psi^{o}, \varphi^{o}, t\right)
$$


3. Sign and zeroes of $b$. There exists a continuous map

$$
Z_{b}:\left[0, \frac{\pi}{2}\right] \rightarrow\left(-\frac{\pi}{2}, 0\right]
$$

continuously differentiable on the open interval $\left(0, \frac{\pi}{2}\right)$, such that

$$
Z_{b}(0)=-\bar{\sigma}
$$

and

$$
\left.\begin{array}{rl}
b(\psi, \varphi)=0, \\
\varphi \geq 0
\end{array}\right\} \Leftrightarrow \begin{cases}\text { either } & \psi=Z_{b}(\varphi) \\
\text { or } & \psi=\pi+Z_{b}(\varphi), \\
\text { or } & \varphi=0 \text { and } \psi \in[-\bar{\sigma}, \bar{\sigma}] \cup[\pi-\bar{\sigma}, \pi+\bar{\sigma}] .\end{cases}
$$

Furthermore,

$$
\left.\begin{array}{r}
b(\psi, \varphi)>0, \\
\varphi \geq 0
\end{array}\right\} \Leftrightarrow \begin{cases}\text { either } & \varphi>0 \text { and } Z_{b}(\varphi)<\psi<\pi+Z_{b}(\varphi), \\
\text { or } & \varphi=0 \text { and } \bar{\sigma}<\psi<\pi+\bar{\sigma},\end{cases}
$$

4. Sign and zeroes of a. One has

$$
\begin{aligned}
& 0<\varphi<\frac{\pi}{2} \Rightarrow a\left(Z_{b}(\varphi), \varphi\right)>0, \\
& a(0,0)=0, \\
\text { and, if } \bar{\sigma}>0, & -\bar{\sigma} \leq \psi<0 \Rightarrow a(\psi, 0)>0 .
\end{aligned}
$$

5. Hyperbolic saddle point at $(\mathbf{0}, \mathbf{0})$. The maps a and $b$ are smooth in a neighborhood of $(0,0)$ and

$$
a(0,0)=b(0,0)=0, \quad \frac{\partial a}{\partial \psi}(0,0) \frac{\partial b}{\partial \varphi}(0,0)-\frac{\partial a}{\partial \varphi}(0,0) \frac{\partial b}{\partial \psi}(0,0)<0 .
$$

6. Values of c at equilibria.

$$
c(0,0)=1, \quad c(\pi, 0)=-1 .
$$

7. Stable and unstable manifolds of $(0,0)$. There exists continuous maps

$$
S:\left[0, \frac{\pi}{2}\right] \rightarrow[-\pi, 0], U:\left[0, \frac{\pi}{2}\right] \rightarrow[0, \pi],
$$

continuously differentiable on the open interval $\left(0, \frac{\pi}{2}\right)$, and a number $\bar{\sigma}$ with

$$
U(0)=0, S(0)=-\bar{\sigma}, \bar{\sigma} \geq 0,
$$

such that the stable and unstable manifolds of $(0,0)$ are described by

$$
\begin{aligned}
& \mathcal{S}^{0}=\left\{(S(\varphi), \varphi), 0 \leq \varphi<\frac{\pi}{2}\right\} \cup[-\bar{\sigma}, \bar{\sigma}] \times\{0\} \cup\left\{(-S(-\varphi), \varphi),-\frac{\pi}{2}<\varphi \leq 0\right\} \\
& \mathcal{U}^{0}=\left\{(U(\varphi), \varphi), 0 \leq \varphi<\frac{\pi}{2}\right\} \cup\left\{(-U(-\varphi), \varphi),-\frac{\pi}{2}<\varphi \leq 0\right\}
\end{aligned}
$$

Furthermore, the zeroes of $b$ are positioned with respect to the stable and unstable manifolds so that the maps $S, U, Z_{b}$ satisfy:

$$
0<\varphi<\frac{\pi}{2} \Rightarrow S(\varphi)<Z_{b}(\varphi)<0<U(\varphi) .
$$

\section{Proof See Appendix A.}

The following theorem is almost independent of the rest of the paper: it states that for any $a, b, c$ that satisfy the seven conditions established in Proposition 5, the differential equation (54) has some properties (that will lead to geodesic convexity); the conditions are of course much more general than the two cases considered in Proposition 5 Theorem 2 will be easily deduced from Theorem 3.

Theorem 3 If a, b, c satisfy the properties of Proposition 5 i.e. (53) through (62), then, for any $\varphi^{0}$ and $\varphi^{1}$ in the interval $(-\pi / 2, \pi / 2)$ and any $\bar{\lambda} \in \mathbb{R}$, there exists $\tau_{\mathrm{fin}} \geq 0$ and a solution $(\psi(),. \varphi()):.\left[0, \tau_{\mathrm{fin}}\right] \rightarrow \mathcal{C}$ of (54) such that

$$
\varphi(0)=\varphi^{0}, \quad \varphi\left(\tau_{\text {fin }}\right)=\varphi^{1}, \quad \int_{0}^{\tau_{\text {fin }}} c(\psi(\tau), \varphi(\tau)) \mathrm{d} \tau=\bar{\lambda} .
$$


Proof of Theorem 3 . See Appendix B.

Proof of Theorem 2 Pick $n^{0}, e^{0}, n^{1}, e^{1}$; according to Proposition 5 Theorem 3 applies to $a, b, c$ defined either by (45) or by (46). Take

$$
\varphi^{0}=\arcsin e^{0}, \varphi^{1}=\arcsin e^{1}, \quad \bar{\lambda}=-\frac{1}{3} \ln \frac{n^{1}}{n^{0}}
$$

and apply this theorem. Use (52) to get $n(\tau)$ and (47) or (48) to get $\rho(\tau)$ (with some arbitrary $\rho(0)$, for instance $\rho(0)=1$ ) and finally (25) to get $e(\tau), p_{n}(\tau), p_{e}(\tau)$ from $\psi(\tau), \varphi(\tau), n(\tau), \rho(\tau)$. Apply the time reparametrization $(\tau \rightsquigarrow t)$ given by (49) or (50), $T$ being deduced from $\tau_{\text {fin }}$ in the same way. According to Proposition 4 the obtained $t \mapsto\left(n(t), e(t), p_{n}(t), p_{e}(t)\right)$ satisfies the conclusions of Theorem 2

\subsection{Simulations}

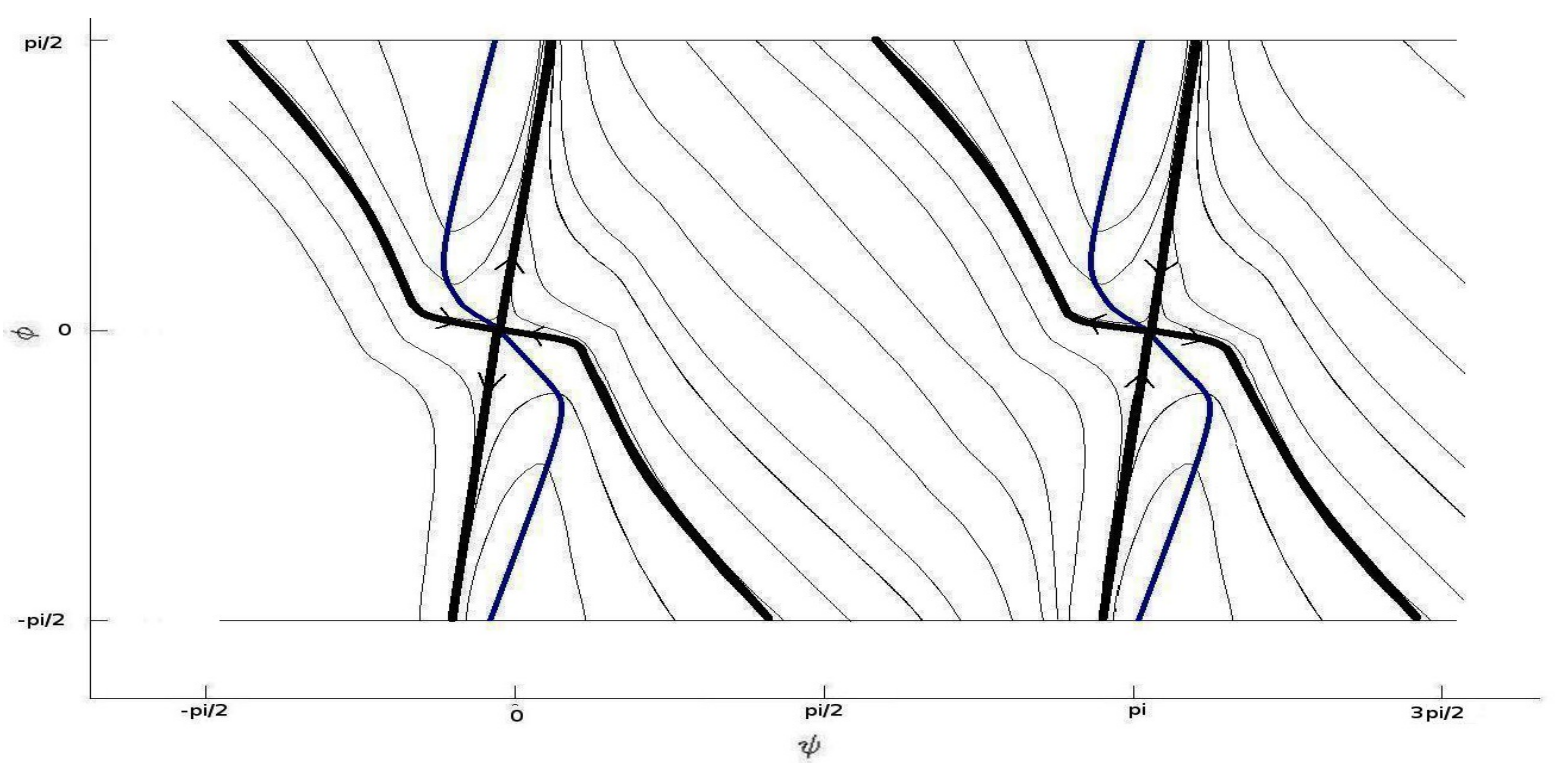

Fig. 1 Numerical plot (obtained using Matlab) of the stable and unstable manifolds (bold) and trajectories through a number of arbitrary initial values in $\mathcal{C}$ for the full control case. The other curve shown is $\psi=Z_{b}(\varphi)$.

A numerical simulation of the phase portrait of the differential equation (54) (or the first two equations in (44)) is displayed in Figure 1 in the "full control case" where $a$ and $b$ are given by (45) and in Figure 2 in the "tangential thrust case" where $a$ and $b$ are given by [46]. This is supposed to be a phase portrait on the cylinder $\mathcal{C}$ (for instance, identify $\left\{\psi=\frac{\pi}{2}\right\}$ with $\left\{\psi=\frac{3 \pi}{2}\right\}$ ).

The thick trajectories are the stable and unstable manifolds of $(0,0)$ and $(\pi, 0)$; the other thick curve is the set of zeroes of $b(\psi, \varphi)$ (i.e. the isocline $\{\dot{\varphi}=0\}$ ). One may check visually the properties established in Proposition 5 . in particular the unstable manifold of $(0,0)$ is, in both cases, a graph $\varphi \mapsto \psi$ while the stable manifold is also such a graph in the full control case (Figure 1) but not in the tangential thrust case (Figure 1) where it comprises a segment of the $\psi$-axis.

It can be seen that in both cases, the cylinder $\mathcal{C}$ is divided into six regions by these invariant manifolds: one region (called $F$ in Appendix B) where all trajectories go "up" ( $\varphi$ is monotone increasing), one (called $F^{+}$in Appendix B where all trajectories go "down", and four other regions (called $E, E^{\sharp}, E^{+}$and $E^{+\sharp}$ in Appendix B where all trajectories cross once the isocline $\{\dot{\varphi}=0\}$ so that they go up and then down or down and then up.

This is exploited in the proof of Theorem 3 . The generic figure 7 is a drawing used to support that proof, that figures in an illustrative manner the features contained in the assumptions of Theorem 3 , and that can also be observed in the numerical simulations of the two cases that we are really interested in (Theorem 2). 


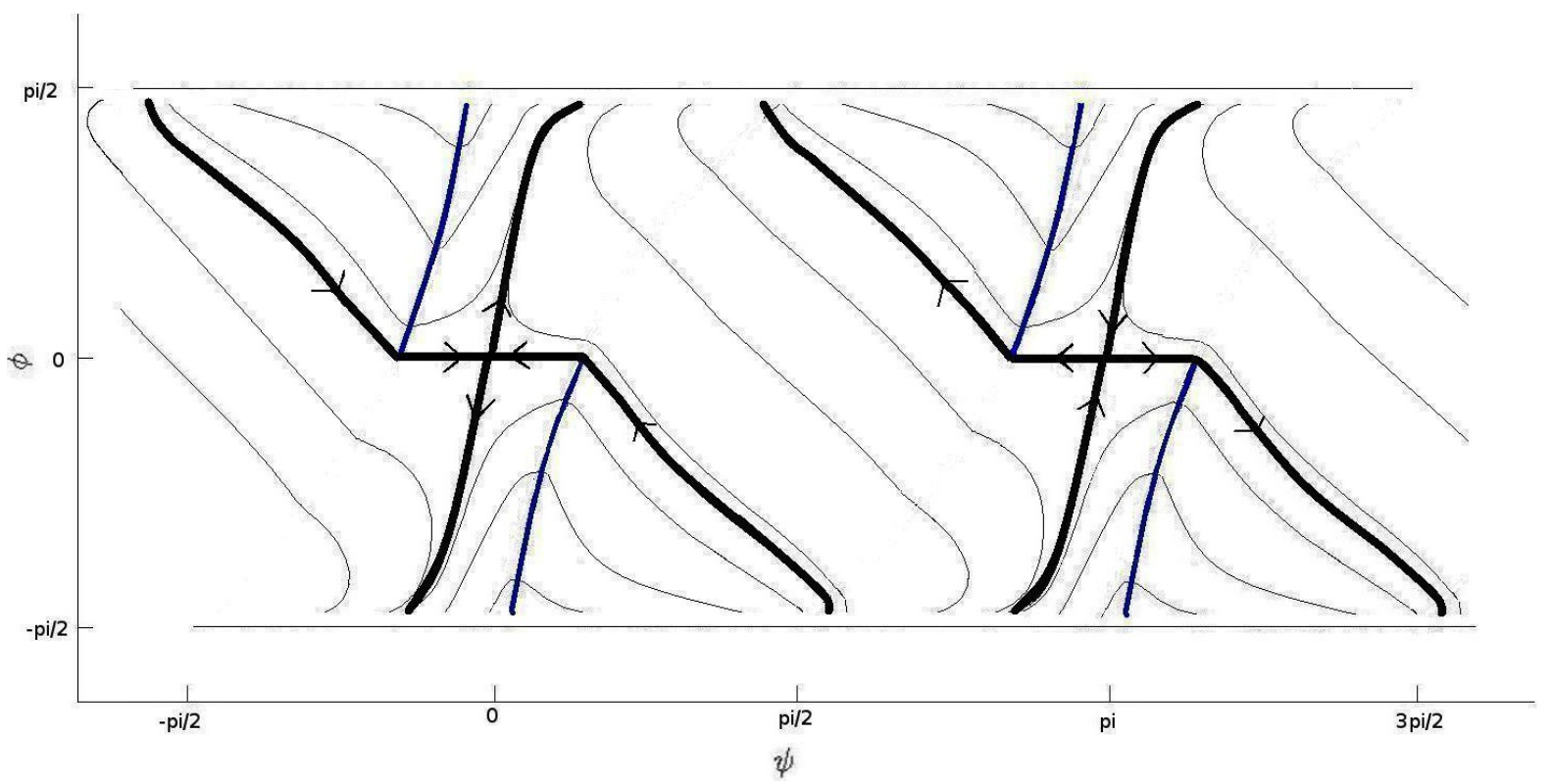

Fig. 2 Numerical plot (obtained using Matlab) of the stable and unstable manifolds (bold) and trajectories through a number of arbitrary initial values in $\mathcal{C}$ for the tangential case. The other curve shown is $\psi=Z_{b}(\varphi)$.

\section{Comparison between the minimum-energy and minimum-time cases from the convexity point of view}

In section 3 we recalled some results from [4] (and previous work by the same authors); in particular, Theorem 1 ] states that the elliptic domain is not geodesically convex for the energy minimization problem, i.e. some pairs of points in $\mathcal{E}$ cannot be joined by a geodesic. In that case, in suitable coordinates $\left(\left(n^{5 / 6}, \sqrt{5 / 2} \varphi\right)\right.$ as polar coordinates), geodesics are straight lines hence geodesic convexity reduces to usual (affine) convexity, thus the simplest way to see this non convexity is to determine the shape on the elliptic domain in these polar coordinates.

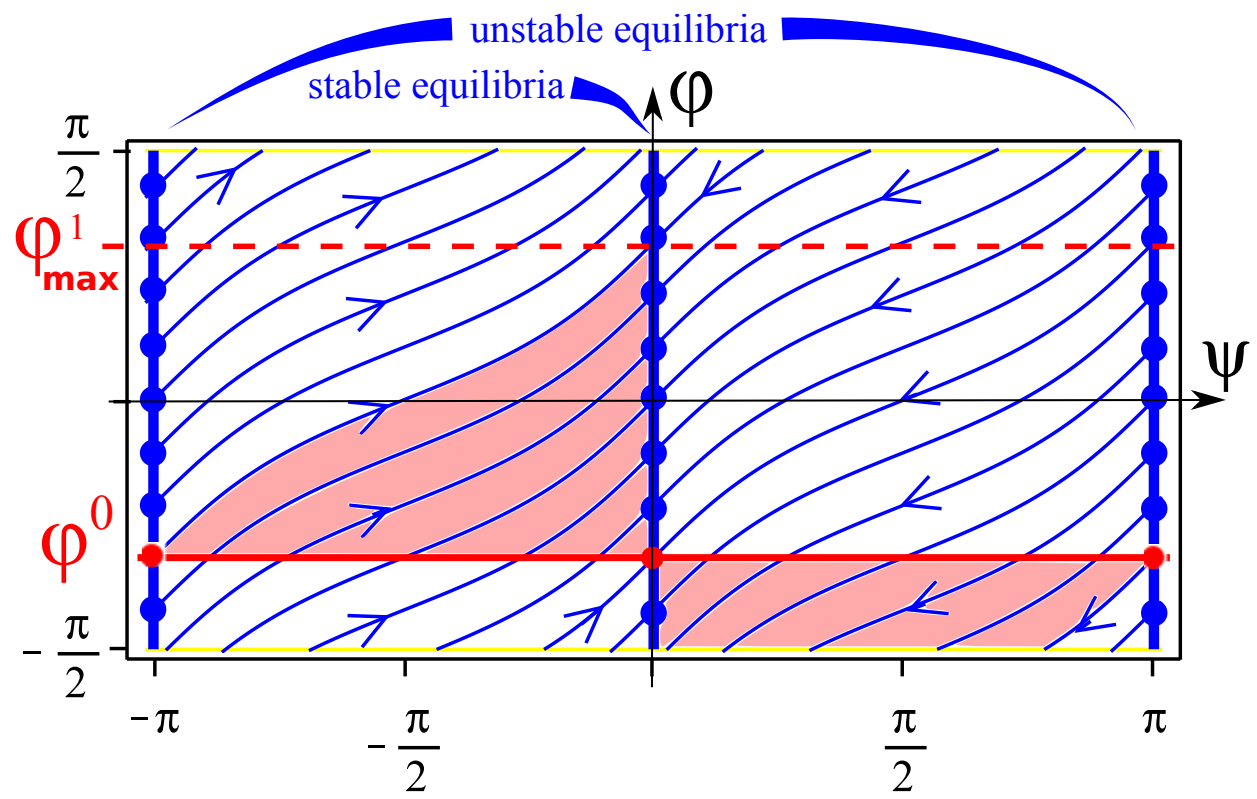

Fig. 3 The phase portrait, for energy minimization, in the same coordinates as Figure 1 and 2 There are two lines of non isolated equilibria. The darker zone is all the points that can be reached in positive time from the line $\left\{\varphi=\varphi^{0}\right\}$, with $\varphi^{0}$ rather close to $-\frac{\pi}{2}$. The highest possible final value is $\varphi$ is $\varphi_{\max }^{1}=\varphi^{0}+\sqrt{2 / 5} \pi$. 
Here we try to explain why convexity holds in the minimum-time case and not in the minimum-energy case. Using the coordinates from Theorem 1 for the time-minimizing problem does not seem to shed any light. Rather, we explain how the proof of convexity that we made in the minimum-time case fails when applied to the minimumenergy case.

When $p_{\omega}=0$, the Hamiltonian in the minimum-energy case is given by (20) and can be written as follows

$$
H=n^{-5 / 3}\left[2\left(3 n p_{n}\right)^{2}+5 p_{\varphi}^{2}\right]
$$

in the coordinates $\left(n, \varphi, p_{n}, p_{\varphi}\right)$ that result from the symplectic change of coordinates $e=\sin \varphi, p_{\varphi}=\sqrt{1-e^{2}} p_{e}$. The Hamiltonian equations can be written

$$
\begin{aligned}
\dot{n} & =12 n^{-2 / 3}\left(3 n p_{n}\right), & \frac{\mathrm{d}}{\mathrm{d} t}\left(3 n p_{n}\right) & =5 n^{-5 / 3}\left[2\left(3 n p_{n}\right)^{2}+5 p_{\varphi}^{2}\right], \\
\dot{\varphi} & =10 n^{-5 / 3} p_{\varphi}, & \dot{p}_{\varphi} & =0 .
\end{aligned}
$$

With the same polar coordinates as in 25) (namely $\rho \cos \psi=3 n p_{n}, \rho \sin \psi=-p_{\varphi}$ ), and the time reparametrization $\mathrm{d} t=5 n^{-5 / 3} \mathrm{~d} \tau$, the state equations of these Hamiltonian equations have the form

$$
\mathrm{d} \psi / \mathrm{d} \tau=-\sin \psi\left(2+3 \sin ^{2} \psi\right), \mathrm{d} \varphi / \mathrm{d} \tau=2 \sin \psi .
$$

It is easy to describe the solutions of these equations on the cylinder $\mathcal{C}$ (see (28)). There are two lines of equilibria at $\psi=0$ and $\psi=\pi$ and

$$
\varphi+\sqrt{\frac{2}{5}} \arctan \left(\sqrt{\frac{5}{2}} \tan \psi\right)
$$

is a first integral (it is smooth at $\psi=\frac{\pi}{2}$ ). These solutions are drawn on Figure 3 It is clear that, on a solution, the maximum possible variation of the variable $\varphi$ is $\sqrt{2 / 5} \pi$; this implies that, if $\left|\varphi^{0}\right|>\left(\sqrt{2 / 5}-\frac{1}{2}\right) \pi$, there are some values of $\varphi$ that cannot be reached by any solution starting from the line $\left\{\varphi=\varphi^{0}\right\}$.

\section{Conclusion and open problems}

We have studied the average minimum time problem as described in section 4.1. This is a reduced subproblem of the planar transfer problem: the state has dimension 2, whereas it would have dimension 3 in the real planar problem (we have set $p_{\omega}=0$; this imposes that the cyclic variable is constant along transfers) and dimension 5 in the full problem where the plane containing the orbits is not fixed.

In [4,5], the energy problem in full dimension is treated; the planar case is integrable (but only the reduced planar case is flat); the full problem is not integrable but extremals may still be computed explicitly. Studying minimum time in higher dimension is an interesting program.

Concerning the reduced problem considered here, the main contribution of the paper is to prove geodesic convexity of the elliptic domain (any two points in the domain may be joined by an extremal trajectory). On the one hand, it is not clear that this result holds true in higher dimension, and on the other hand, in the present small dimension, optimality and/or uniqueness of the extremal trajectories has not been studied.

Finally the singularities of the Hamiltonian have been investigated roughly, mostly to ensure existence of a Hamiltonian flow. It would be interesting to better understand their nature and their role, in particular the singularities they cause on the balls of small radius for the metric.

\section{APPENDIX}

\section{A Proof of Proposition 5}

Let us prove that the seven points in Proposition 5 are satisfied by $a, b, c$ given by (45) (full control case) and also by $a, b, c$ given by (46) (tangential thrust case).

11. Symmetries. Equations (30) and 31 imply

$$
\widetilde{I}(\pi+\psi, \varphi, E)=-\widetilde{I}(\psi, \varphi, E), \quad \widetilde{I}(-\psi,-\varphi, \pi-E)=\widetilde{I}(\psi, \varphi, E)
$$


while 33 implies

$$
\widetilde{J}(\psi+\pi, \varphi, E)=-\widetilde{J}(\psi, \varphi, E), \quad \widetilde{J}(-\psi,-\varphi, \pi-E)=\widetilde{J}(\psi, \varphi, E) .
$$

Substituting in 29) and (32) yields, using the change of variable $E \rightarrow \pi-E$ in the integrals $L(-\psi,-\varphi)$ and $M(-\psi,-\varphi)$,

$$
L(\pi+\psi, \varphi)=L(-\psi,-\varphi)=L(\psi, \varphi), M(\pi+\psi, \varphi)=M(-\psi,-\varphi)=M(\psi, \varphi) .
$$

This yields identities (53) with $a, b, c$ given either by (45) or by (46).

2 Uniqueness of solutions. This follows from the classical Cauchy-Lipschitz theorem away from $\mathcal{S}$ (see Proposition 3. On $\mathcal{S}$,

- in the full control case ( $a, b$ given by (45)), as seen in [3], the regularity properties (35) of the right hand side of (54) guarantee the existence and uniqueness of solutions to the Cauchy problem (Kamke uniqueness Theorem [13, chap. III, Th.6.1]),

- in the tangential thrust case ( $a, b$ given by (46), the same argument does not apply but one may check that the derivative of $\tan \psi-\frac{1}{2}( \pm 1+\sin \varphi) / \cos \varphi$ along $\dot{\psi}=a(\psi, \varphi), \dot{\varphi}=b(\psi, \varphi)$ is nonzero along the curve $\tan \psi=\frac{1}{2}( \pm 1+\sin \varphi) / \cos \varphi$, hence the vector field is transverse to $\mathcal{S}$ and this implies uniqueness of solutions starting from a point in $\mathcal{S}$ (see e.g. [10]).

Continuity of $\Phi$ in (55), is, according to [13, chap. V, Theorem 2.1], guaranteed by uniqueness of solutions and continuity of $a, b$.

3. Sign and zeroes of $b$.

3. Full control case ( $a, b$ given by (45)). On the one hand, one has

$$
b(0, \varphi)=\frac{2 \cos \varphi \sin \varphi}{\pi} \int_{0}^{\pi} \frac{\cos ^{2} E \mathrm{~d} E}{\sqrt{1-\sin ^{2} \varphi \cos ^{2} E}}, \quad b(-\pi, \varphi)=-b(0, \varphi) .
$$

On the other hand, the derivative of $b(\psi, \varphi)$ with respect to $\psi$ is given by

$$
\frac{\partial b}{\partial \psi}(\psi, \varphi)=\frac{\cos \psi}{\pi} \int_{0}^{\pi} \frac{\left(1-\sin ^{2} \varphi \cos ^{2} E\right)^{4} \sin ^{2} E}{\widetilde{I}(\psi, \varphi, E)^{3 / 2}} \mathrm{~d} E .
$$

The integrals in both equations are positive. Hence for any fixed $\varphi \geq 0, b(\psi, \varphi)$ is increasing with respect to $\psi$ on $\left(-\frac{\pi}{2}, \frac{\pi}{2}\right)$ and decreasing on $\left(\frac{\pi}{2}, \frac{3 \pi}{2}\right)$; according to $(71)$, it is positive in $\left(0, \frac{\pi}{2}\right]$ and negative in $\left(\pi, \frac{3 \pi}{2}\right]$ (identified with $\left.\left(-\pi,-\frac{\pi}{2}\right]\right)$, hence it must vanish for a unique value of $\psi$ between $-\frac{\pi}{2}$ and 0 , that we call $Z_{b}(\psi)$, thus defining $Z_{b}:\left[0, \frac{\pi}{2}\right) \rightarrow\left(-\frac{\pi}{2}, 0\right]$. It also vanishes for a unique value of $\psi$ between $\frac{\pi}{2}$ and $\pi$ that must be equal to $\pi+Z_{b}(\psi)$ according to (53). According to (71), $Z_{b}(0)=0$ and $Z_{b}(0)<0$ if $\varphi>0$.

Fig. 4 Numerical plot of the function $\sqrt{73}$ on the interval $[0,1)$. Obtained with Maple 15.

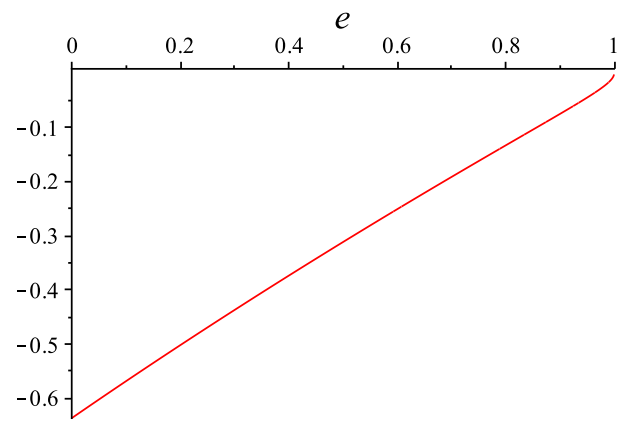

Proposition 3 says that $L$, and hence $b$, are smooth away from $\mathcal{S}$. The part of $\mathcal{S}$ that is contained in the square $\left(-\frac{\pi}{2}, 0\right] \times\left[0, \frac{\pi}{2}\right)$ is the curve $\left\{\tan \psi=\frac{-1+\sin \varphi}{2 \cos \varphi}, 0 \leq \varphi<\frac{\pi}{2}\right\}$. We claim that $\gamma(\varphi)=b\left(\arctan \frac{-1+\sin \varphi}{2 \cos \varphi}, \varphi\right)$ does not vanish between 0 and $\frac{\pi}{2}$; this is numerically checked by plotting, on Figure 4, the graph of the function $e \mapsto \gamma(\arcsin e)$ on $[0,1]$, i.e.

$$
e \mapsto b\left(\arctan \frac{-1+e}{2 \sqrt{1-e^{2}}}, \arcsin e\right)
$$


On the one hand, this proves that $b$ is smooth at points where it vanishes; on the other hand the derivative of $b$ with respect to $\psi$ is (see (72)) strictly positive at $\left(Z_{b}(\varphi), \varphi\right), \varphi>0$. This implies smoothness of $Z_{b}$ according to the inverse function theorem; and this extends to negative $\varphi$ with $Z_{b}(0)=0$, hence point 3 of the proposition is satisfied with $\bar{\sigma}=0$; it is also easy to check that $b(\psi, 0)$ only if $\psi=0$ or $\psi=\pi$.

3. Tangential thrust case ( $a, b$ given by (46)). In the region $\mathcal{R}_{1}$, one has

$$
b(\psi, \varphi)=(\operatorname{sign} \cos \psi) \frac{2 \cos \varphi \sin \varphi}{\pi} \int_{0}^{\pi} \frac{\cos ^{2} E}{\sqrt{1-\sin ^{2} \varphi \cos ^{2} E}} \mathrm{~d} E .
$$

The derivative of $b(\psi, \varphi)$ with respect to $\psi$ is zero in $\mathcal{R}_{1}$ because the above does not depend on $\psi$, and in $\mathcal{R}_{2}$ it is given by

$$
b_{\psi}(\psi, \varphi)=\frac{\operatorname{sign}(P(\psi, \varphi)) 8 \cos ^{2} \varphi R(\psi, \varphi)(1-R(\psi, \varphi) \sin \varphi)}{\sqrt{1-R(\psi, \varphi)^{2}} \sqrt{1-R(\psi, \varphi)^{2} \sin ^{2} \varphi}(2 \sin \varphi \cos \psi-\cos \varphi \sin \psi)^{2}}
$$

Since $|R|<1$ on $\mathcal{R}_{2}$, all factors are positive except $R(\psi, \varphi)$, hence $b_{\psi}$ vanishes in $\mathcal{R}_{2}$ at points where $R$ vanishes, and this is exactly, according to 39 , on the lines $\left\{\psi= \pm \frac{\pi}{2}\right\}$, so that $b_{\psi}(\psi, \varphi)$ has the sign of $P(\psi, \varphi) R(\psi, \varphi)$, i.e. of $\cos \psi$. Hence, for fixed $\varphi \geq 0, b(\psi, \varphi)$ is

- minimum and negative for $\psi=-\pi / 2$,

- increasing for $\psi$ in $\left(-\frac{\pi}{2}, \arctan \left(\frac{-1+\sin \varphi}{2 \cos \varphi}\right)\right)$,

- constant, positive if $\varphi>0$ and zero if $\varphi=0$, for $\psi$ in $\left[\arctan \left(\frac{-1+\sin \varphi}{2 \cos \varphi}\right), \arctan \left(\frac{1+\sin \varphi}{2 \cos \varphi}\right)\right]$,

- increasing, hence positive, for $\psi$ in $\left(\arctan \left(\frac{1+\sin \varphi}{2 \cos \varphi}\right), \frac{\pi}{2}\right)$,

- maximum and positive for $\psi=\frac{\pi}{2}$.

Hence, for any $\varphi>0$, there is a unique $\psi,-\frac{\pi}{2}<\psi<\arctan \left(\frac{-1+\sin \varphi}{2 \cos \varphi}\right)$ such that $b(\psi, \varphi)=0$; we call it $Z_{b}(\varphi)$, thus defining $Z_{b}:\left(0, \frac{\pi}{2}\right) \rightarrow\left(-\frac{\pi}{2}, 0\right)$, satisfying $-\frac{\pi}{2}<Z_{b}(\varphi)<\arctan \left(\frac{-1+\sin \varphi}{2 \cos \varphi}\right)$ satisfying (58)-(59) (situation on $\left[\frac{\pi}{2}, \frac{3 \pi}{2}\right]$ by symmetry, see $[53)$ ).

Since (74) is valid also on $\mathcal{S}$ by continuity, $b$ does not vanish on $\mathcal{S}$ except at $\varphi=0$, hence $b$ is smooth when it vanishes, away from $\varphi=0$; since we also proved that $b_{\psi}$ is nonzero at these points, the inverse function theorem implies that $Z_{b}$ is smooth on the open interval $\left(0, \frac{\pi}{2}\right)$; also the monotonicity argument shows that $\lim _{\varphi \rightarrow 0} Z_{b}(\varphi)=$ $-\arctan \frac{1}{2}$, hence $Z_{b}$ is defined $\left[0, \frac{\pi}{2}\right) \rightarrow\left(-\frac{\pi}{2}, 0\right)$ with $Z_{b}(0)=-\arctan \frac{1}{2}$. Since our considerations above for $\varphi \geq 0$ imply that $b(0, \varphi)$ is zero if and only if $\psi \in\left[-\arctan \frac{1}{2}, \arctan \frac{1}{2}\right] \cup\left[\pi-\arctan \frac{1}{2}, \pi+\arctan \frac{1}{2}\right]$, we have proved point 3 of the proposition with $\bar{\sigma}=\arctan \frac{1}{2}$.

4. Sign and zeroes of $a$. In 60, the part saying that $a(\psi, 0)>0$ for $-\bar{\sigma} \leq \psi<0$ needs no proof in the full control case because $\bar{\sigma}=0$ and is easy in the tangential thrust case because, from (40) and (46), $a(\psi, 0)=$ $(1+\cos \psi) \sin \psi$ in $\mathcal{R}_{2}$.

We give numerical evidence that $a\left(Z_{b}(\varphi), \varphi\right)$ is positive if $0<\varphi<\frac{\pi}{2}$. Note that the map $Z_{b}$ can only be determined numerically as the zero $\psi=Z_{b}(\varphi)$ of $b(\psi, \varphi)=0$ between $-\frac{\pi}{2}$ and 0 for fixed $\varphi$, but the determination is very reliable for $b$ is monotonous with respect to $\psi=Z_{b}(\varphi)$ in the considered region; see point 3 .

Figure 5 displays a numerical plot of the graph of the map $\varphi \mapsto a\left(Z_{b}(\varphi), \varphi\right)$ in the full control case; we also show $\varphi \mapsto Z_{b}(\varphi)$. Figure 6 displays a numerical plot of the graph of $\varphi \mapsto a\left(Z_{b}(\varphi), \varphi\right)$ in the tangential thrust case; we also show $\varphi \mapsto Z_{b}(\varphi)$ and $\varphi \mapsto \arctan \left(\frac{-1+\sin \varphi}{2 \cos \varphi}\right)$ to show that it is very close to $Z_{b}\left(\psi=\arctan \left(\frac{-1+\sin \varphi}{2 \cos \varphi}\right)\right.$ is the curve where $R(\psi, \varphi)=-1$, the border between $\mathcal{R}_{1}$ and $\mathcal{R}_{2}$ ).

5. Hyperbolic saddle. Smoothness around the origin follows from Proposition 3 . It is clear in both cases that $a(0,0)=0, b(0,0)=0$. The computation of the Jacobians is easy (in the tangential thrust case it takes place in the region $\mathcal{R}_{1}$ with $\cos \psi \geq 0$, see $(74)$ ).

In the full control case,

$$
\frac{\partial a}{\partial \psi}(0,0)=-2, \frac{\partial a}{\partial \varphi}(0,0)=\frac{1}{2}, \frac{\partial b}{\partial \psi}(0,0)=\frac{1}{2}, \frac{\partial b}{\partial \varphi}(0,0)=1 .
$$

The eigenvalues of the Jacobian $\left(\begin{array}{cc}-2 & 1 / 2 \\ 1 / 2 & 1\end{array}\right)$ are $(\sqrt{10}-1) / 2$ (unstable) and $-(\sqrt{10}+1) / 2$ (stable) associated to the eigenvectors $(\sqrt{10}-3,1)$ and $(-\sqrt{10}-3,1)$, respectively. 
Fig. 5 Plots (obtained with Matlab) of the maps $\varphi \mapsto Z_{b}(\varphi)$ (dashed line) and $\varphi \mapsto a\left(Z_{b}(\varphi), \varphi\right)$ (solid line) in the full control case.
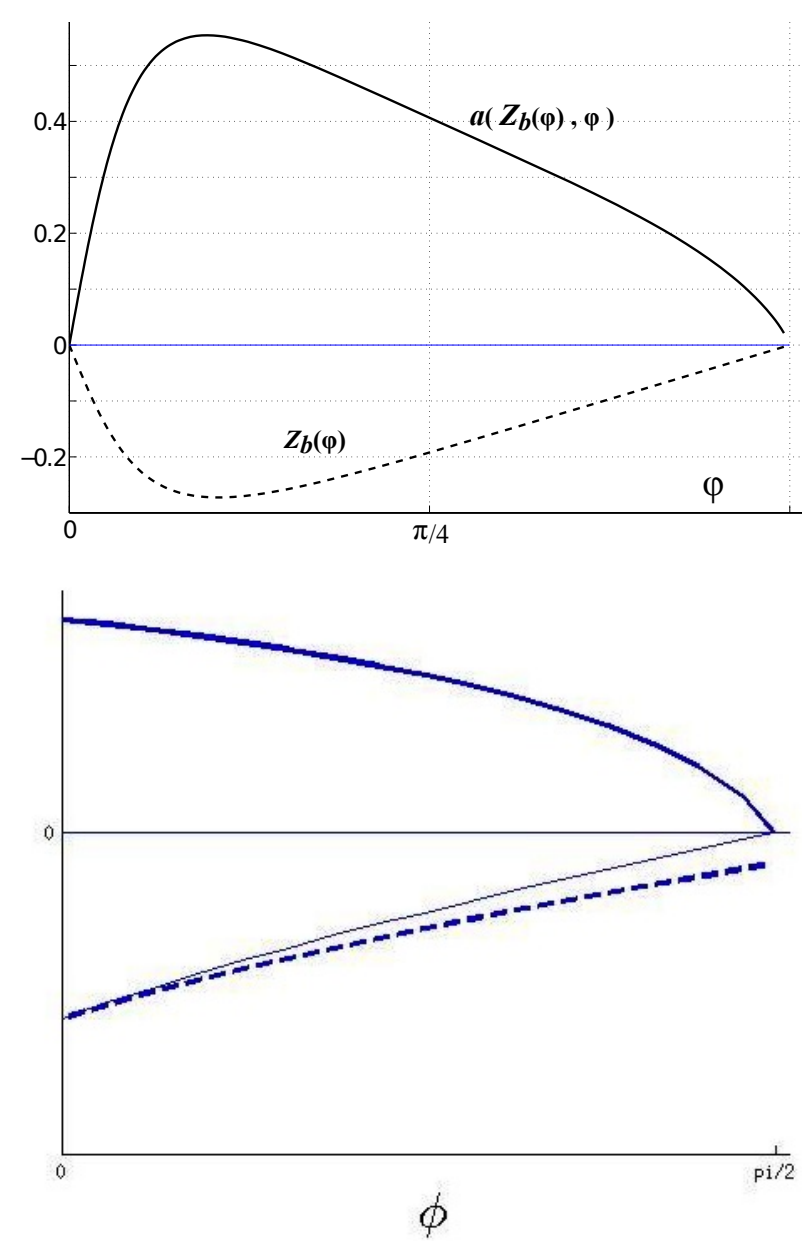

Fig. 6 The plots (obtained with Matlab) of the functions $\varphi \mapsto Z_{b}(\varphi)$ (dashed) and $\psi=a\left(Z_{b}(\varphi), \varphi\right)$ for $\varphi \in[0, \pi / 2]$. Note that $a\left(Z_{b}(\varphi), \varphi\right)$ is everywhere positive on this interval. The other curve shown is the curve $R(\psi, \varphi)=-1$.

$$
\frac{\partial a}{\partial \psi}(0,0)=-2, \frac{\partial a}{\partial \varphi}(0,0)=\frac{1}{2}, \frac{\partial b}{\partial \psi}(0,0)=0, \frac{\partial b}{\partial \varphi}(0,0)=1 .
$$

The eigenvalues of the Jacobian $\left(\begin{array}{cc}-2 & 1 / 2 \\ 0 & 1\end{array}\right)$ at $(0,0)$ are 1 (unstable) and -2 (stable), associated to the eigenvectors $(1 / 6,1)$ and $(1,0)$, respectively.

6. Values of c at equilibria. One deduces $L(0,0)=L(\pi, 0)=1$ from 29, , 30, , 31) and $M(0,0)=M(\pi, 0)=1$ from (32), 33). According to (45) and (46), this implies $c(0,0)=1, c(0, \pi)=-1$ in both cases.

7. Stable and unstable manifolds of $(0,0)$.

71 full control case. The unstable manifold is the union of the equilibrium $(0,0)$ and two solutions that tend to $(0,0)$ as time $\tau$ tends to $-\infty$ and are, according to $(76)$, both tangent to the line $\{\psi=(\sqrt{10}-3) \varphi\}$ at $(0,0)$. One of the solutions approaches with positive $\psi$ and $\varphi$ and the other with negative $\psi$ and $\varphi$. We consider only the first one and call it $\tau \mapsto(\bar{\psi}(\tau), \bar{\varphi}(\tau))$, defined on the time interval $\left(-\infty, \bar{\tau}^{+}\right), \bar{\tau}^{+} \leq+\infty$; the result for the other one follows by symmetry. Let $\mathcal{D}_{1}$ be the rectangle

$$
\mathcal{D}_{1}=\{(\psi, \varphi) \in \mathcal{C}, 0<\psi<\pi, 0<\varphi\} .
$$

On the one hand, we have $\lim _{\tau \rightarrow-\infty} \bar{\psi}(\tau)=\lim _{\tau \rightarrow-\infty} \bar{\varphi}(\tau)=0, \lim _{\tau \rightarrow-\infty}(\bar{\psi}(\tau) / \bar{\varphi}(\tau))=\sqrt{10}-3$, hence $(\bar{\psi}(\tau), \bar{\varphi}(\tau)) \in \mathcal{D}_{1}$ for $\tau$ close enough to $-\infty$. On the other hand, the border of $\mathcal{D}_{1}$ is made of the two equilibria and three segments

$$
\left\{\psi=0,0<\varphi<\frac{\pi}{2}\right\},\left\{\psi=\pi, 0<\varphi<\frac{\pi}{2}\right\},\{0<\psi<\pi, \varphi=0\} .
$$

A short computation shows that

$$
a(0, \varphi)=\frac{\cos \varphi \sin \varphi}{\pi} \int_{0}^{\pi} \frac{\cos ^{2} E \mathrm{~d} E}{\sqrt{1-\sin ^{2} \varphi \cos ^{2} E}}, \quad a(\pi, \varphi)=-a(0, \varphi),
$$


hence $a$ is positive on the first segment and negative on the second one; according to the proof of Point 3 above, $b$ is positive on the last one; hence solutions starting on these segments all enter $\mathcal{D}_{1}$. This proves positive invariance of $\mathcal{D}_{1}$ (solutions may "exit" through the segment $\left\{\varphi=\frac{\pi}{2}\right\}$, but they are no longer defined). Hence the solution remains in $\mathcal{D}_{1}$ for all time in the open interval $\left(-\infty, \bar{\tau}^{+}\right)$. According to Point $3, b(\psi, \varphi)$ is positive on $\mathcal{D}_{1}$. This solution cannot remain in a compact subset of $\mathcal{C}$ for all time because then it would have a non-empty $\omega$-limit set that would have to be a union of equilibria and periodic solutions by Poincaré-Bendixon Theorem, but the fact that $\dot{\varphi}>0$ in $\mathcal{D}_{1}$ prevents periodic solutions from existing and the only equilibria are $(0,0)$ and $(\pi, 0)$, that cannot be approached because $\bar{\varphi}(t)$ cannot become small for $\bar{\varphi}(\tau)$ is increasing. Hence necessarily, $\lim _{t \rightarrow \bar{\tau}}+\bar{\varphi}(t)=\frac{\pi}{2}$. We have established that the parametrized curve $t \mapsto(\bar{\psi}(t), \bar{\varphi}(t)),-\infty<t<\bar{\tau}^{+}$defines the graph of a function $\psi=U(\varphi),\left(0, \frac{\pi}{2}\right) \rightarrow(0, \pi)$; it is continuously differentiable from the implicit function theorem: since the righthand side of the differential equation is continuous, the parameterized curve is continuously differentiable, and we saw that the derivative of $\varphi$ with respect to the parameter (time) remains positive (again because $b>0$ in $\mathcal{D}_{1}$ ).

Let us turn to the stable manifold. It is the union of the equilibrium $(0,0)$ and two solutions that tend to $(0,0)$ as time $\tau$ tends to $+\infty$. According to the proof of point 5 , both solutions are tangent to the line $\{\psi=-(\sqrt{10}+3) \varphi\}$ at the origin. We consider the solution that approaches $(0,0)$ with negative $\psi$ and positive $\varphi$, and call it $\tau \mapsto$ $(\bar{\psi}(\tau), \bar{\varphi}(\tau))$; the result for the other one follows by symmetry. The proof is now very similar to the one for the unstable manifold, reversing time and replacing $\mathcal{D}_{1}$ by the domain

$$
\mathcal{D}_{2}=\left\{(\psi, \varphi) \in \mathcal{C},-\pi<\psi<Z_{b}(\varphi), \varphi>0\right\}
$$

Firstly, $b(\psi, \varphi)$ is negative in this domain. Secondly, the solution is in this domain for $\tau$ large enough (obviously $\psi$ is negative and $\varphi$ is positive, and it is on the right side of $\psi=Z_{b}(\varphi)$ because $\varphi$ tends to zero so the solution must spend some time in the region where $b<0$ ). Thirdly, the domain $\mathcal{D}_{2}$ is negatively invariant: its border is made of the equilibria, the segments $\left\{\psi=-\pi, 0<\varphi<\frac{\pi}{2}\right\},\{-\pi<\psi<0, \varphi=0\}$ and the curve $\left\{\psi=Z_{b}(\varphi), 0<\varphi<\frac{\pi}{2}\right\}$. Solutions which start on the segments leave $\mathcal{D}_{2}$ because of (78) and the fact that that $b(\psi, 0)<0$ if $-\pi<\varphi<0$ (see Point 3 above). Solutions which start on the curve leave the domain $\mathcal{D}_{2}$ because of Point 4 above (at these points, $Z_{b}$ is differentiable, $\left.Z_{b}(\bar{\psi}(\tau), \bar{\varphi}(\tau))=0, a(\bar{\psi}(\tau), \bar{\varphi}(\tau))>0, b(\bar{\psi}(\tau), \bar{\varphi}(\tau))=0\right)$. This with the second point implies that the solution is in the domain for all time. The end of the proof, i.e. definition of the continuously differentiable $S$ is exactly the same as the previous proof, only with $b<0$ instead of $b>0$. Moreover, we get for free that $-\pi<S(\varphi)<Z_{b}(\varphi)$ from the definition of $\mathcal{D}_{2}$; this and the above implies 66.

7.2 tangential thrust case. Let us first compute some values of $a$ and $b$ on special lines? according to (46) and (32)-(33),

$$
\begin{gathered}
a\left(\frac{\pi}{2}, \varphi\right)=-\frac{2|\cos \varphi|}{\sin \varphi} \ln \left(\frac{1+\sin \varphi}{1-\sin \varphi}\right), \\
a\left(-\frac{\pi}{2}, \varphi\right)=\frac{2|\cos \varphi|}{\sin \varphi} \ln \left(\frac{1+\sin \varphi}{1-\sin \varphi}\right), \\
a(0, \varphi)=\frac{\cos \varphi \sin \varphi}{\pi} \int_{0}^{\pi} \frac{\cos ^{2} E \mathrm{~d} E}{\sqrt{1-\sin ^{2} \varphi \cos ^{2} E}}, \\
b(\psi, 0)= \begin{cases}\operatorname{sign}(\sin \psi) \frac{2}{\pi} \sqrt{4-\cot ^{2} \psi} & \text { if } \left.\tan \psi \geq \frac{1}{2} \text { (i.e. in } \mathcal{R}_{2}\right), \\
0 & \text { if } \left.\tan \psi \leq \frac{1}{2} \text { (i.e. in } \mathcal{R}_{1}\right) .\end{cases}
\end{gathered}
$$

The unstable manifold comprises the equilibrium point $(0,0)$ and two solutions that tend towards it as time $\tau$ tends to $-\infty$, tangent, according to (77), to the line $\{\psi=\varphi / 6\}$ at $(0,0)$. Thus either both $\varphi$ and $\psi$ are positive as they approach $(0,0)$ or they are both negative. Of the two solutions, we will only consider the one where $\psi, \varphi$ are both positive, and call it $\tau \rightarrow(\bar{\psi}(\tau), \bar{\varphi}(\tau))$. Obviously, $(\bar{\psi}(\tau), \bar{\varphi}(\tau))$ is in the rectangle

$$
\mathcal{D}_{1}=\{(\psi, \varphi) \in \mathcal{C}: 0<\psi<\pi / 2, \varphi>0\}
$$

for $\tau$ negative large enough. From Point 32 above, $b(\psi, \varphi)>0$ in the whole of $\mathcal{D}_{1}$. From (79) and $(80), a(0, \varphi)>$ $0, a(\pi / 2, \varphi)<0$, and $b(\psi, 0) \geq 0$ for $0<\psi<\pi, \varphi>0$, thus $\mathcal{D}_{1}$ is positively invariant. The rest of the proof follows exactly the same argument as for the case of the unstable manifold in Point 71 above.

\footnotetext{
2 The journal version unfortunately contains some misprints in equations (79)-(80); they are corrected here.
} 
We now consider the stable manifold. It comprises the equilibrium point $(0,0)$ and two solutions that tend towards it as time $\tau$ tends to $+\infty$. They are both tangent at $(0,0)$ to the stable eigenvector, i.e. (see (77) and the sequel) to the line $\{\varphi=0\}$. Since $\dot{\varphi}$, i.e. $b(\psi, \varphi)$, is zero on the segment $\left\{\varphi=0,-\arctan \frac{1}{2} \leq \psi \leq \arctan \frac{1}{2}\right\}$, these solutions follow this segment. We examine the one that approaches $(0,0)$ with negative $\psi$, the other one follows by symmetry. Call $\tau \mapsto(\bar{\psi}(\tau), \bar{\varphi}(\tau))$ the solution such that $\bar{\psi}(0)=-\arctan \frac{1}{2}, \bar{\varphi}(0)=0$. One has $\bar{\varphi}(\tau)=0$ for all positive $\tau$ and $\bar{\psi}(\tau)$ is increasing for positive $\tau$ and tends to zero as $\tau \rightarrow+\infty$. Define the domain

$$
\mathcal{D}_{2}=\left\{(\psi, \varphi) \in \mathcal{C}:-\frac{\pi}{2}<\psi<Z_{b}(0), \varphi>0\right\}
$$

The solution is outside $\mathcal{D}_{2}$ for positive $\tau$ but on its border at $\tau=0$. From (79), $a\left(-\frac{\pi}{2}, \varphi\right)>0$ for all $\varphi$ between 0 and $\frac{\pi}{2}$; from $(79), b(\psi, 0)<0$ for $\psi \in\left(-\frac{\pi}{2},-\arctan \frac{1}{2}\right)\left(-\arctan \frac{1}{2}\right.$ is $\left.\left.Z_{b}(0)\right)\right)$; from point 4 above, $a\left(Z_{b}(\varphi), \varphi\right)>0$ if $\psi \in\left(0, \frac{\pi}{2}\right)$. Hence $\mathcal{D}_{2}$, as well as its topological closure, are negatively invariant. Since $(\bar{\psi}(0), \bar{\varphi}(0))$ is on the boundary of $\mathcal{D}_{2}$, one has $(\bar{\psi}(\tau), \bar{\varphi}(\tau)) \in \mathcal{D}_{2}$ for all $\tau \in\left(\tau^{-}, 0\right)$ where $\left(\tau^{-},+\infty\right)$ is the maximal interval of definition of the solution we consider. From (59) and (58), $b(\psi, \varphi)<0$ for all $(\psi, \varphi)$ in $\mathcal{D}_{2}$. Then, following the same argument as in the proof concerning the stable manifold in Point 7 1 above, we obtain that the restriction to negative times of the solution is the graph $\varphi=S(\psi)$ where $S:\left[0, \frac{\pi}{2}\right) \rightarrow\left(-\frac{\pi}{2}, 0\right]$ is continuously differentiable on $\left(0, \frac{\pi}{2}\right)$ and $S(0)=-\arctan \frac{1}{2}$. We already noticed that the other part of the solution covers the segment $\left\{\varphi=0,-\arctan \frac{1}{2} \leq \psi<0\right\}$. This ends the proof of point Point 7 (63) to 66) in the tangential thrust case.

\section{B Proof of Theorem 3}

Theorem 3 and this section are independent of the rest of the paper: here we only refer to the seven conditions ranging from equation 53 to equation 66 .

Lemma 1 Assume that $a, b, c$ satisfy (53) (i.e. point 1).

If $\tau \mapsto(\psi(\tau), \varphi(\tau)) \in \mathcal{C}$ is a solution of (54) defined on the time interval $\left[0, \tau_{\text {fin }}\right]$, then $\tau \mapsto\left(\psi^{\sharp}(\tau), \varphi^{\sharp}(\tau)\right)$ and $\tau \mapsto\left(\psi^{+}(\tau), \varphi^{+}(\tau)\right)$ with

$$
\begin{array}{ll}
\psi^{\sharp}(\tau)=-\psi(\tau), & \psi^{+}(\tau)=\psi\left(\tau_{\text {fin }}-\tau\right)+\pi, \\
\varphi^{\sharp}(\tau)=-\varphi(\tau), & \varphi^{+}(\tau)=\varphi\left(\tau_{\text {fin }}-\tau\right),
\end{array}
$$

are also solutions of (54) on the same time interval $\left[0, \tau_{\text {fin }}\right]$ and they satisfy

$$
\int_{0}^{\tau_{\text {fin }}} c\left(\psi^{\sharp}(\tau), \varphi^{\sharp}(\tau)\right) \mathrm{d} \tau=\int_{0}^{\tau_{\text {fin }}} c\left(\psi^{+}(\tau), \varphi^{+}(\tau)\right) \mathrm{d} \tau=\int_{0}^{\tau_{\text {fin }}} c(\psi(\tau), \varphi(\tau)) \mathrm{d} \tau .
$$

Proof This is straightforward.

We also use the " $\sharp "$ and " $+"$ notation to denote the transformations in $\mathcal{C}$ :

$$
(\psi, \varphi)^{\sharp}=(-\psi,-\varphi), \quad(\psi, \varphi)^{+}=(\pi+\psi, \varphi) .
$$

Let us make further constructions and remarks on the conditions [53)- 66 before proceeding with the proof perse.

Stable and unstable manifolds. Equation 61) implies that the Jacobian of the vector field at the equilibrium $(0,0)$ has two real eigenvalues, of which one is positive and the other negative; i.e. $(0,0)$ is an hyperbolic saddle (see e.g. [14, section 8.3]). Thus it has a stable manifold $\mathcal{S}^{0}$ and an unstable manifold $\mathcal{U}^{0}$; these are curves passing through $(0,0)$ tangent to the corresponding eigenvectors. Their existence is a consequence of (61) but point 7 assumes a more specific description. 
The number $\bar{\sigma}$. Everything may be stated in a much simpler if $\bar{\sigma}=0$ : in particular in Points 3 and 7, $S$ and $Z_{b}$ may be continued into continuous even maps $\left(-\frac{\pi}{2}, \frac{\pi}{2}\right) \rightarrow \mathbb{R}$, and, for instance, the equations of $\mathcal{S}^{0}$ and $\mathcal{U}^{0}$, instead of 65), as $\left\{\psi=S(\varphi),-\frac{\pi}{2}<\varphi<\frac{\pi}{2}\right\}$ and $\left\{\psi=U(\varphi),-\frac{\pi}{2}<\varphi<\frac{\pi}{2}\right\}$. We would have preferred this simpler formulation but we do not assume $\bar{\sigma}=0$ because the proof of Theorem 2 in the tangential case uses Theorem 3 with a nonzero $\bar{\sigma}\left(\bar{\sigma}=\arctan \frac{1}{2}\right)$.

However, in order to avoid considering positive and negative $\varphi$ 's as different cases in (65), (58) and (59), we define the functions $U^{0}:\left[-\frac{\pi}{2}, \frac{\pi}{2}\right] \rightarrow[-\pi, \pi], S^{0}:\left[-\frac{\pi}{2}, \frac{\pi}{2}\right] \backslash\{0\} \rightarrow[-\pi, \pi], Z_{b}^{0}:\left[-\frac{\pi}{2}, \frac{\pi}{2}\right] \backslash\{0\} \rightarrow\left(-\frac{\pi}{2}, \frac{\pi}{2}\right)$ after $S$, $U, Z_{b}$; these functions are odd and coincide with the former on $\left(0, \frac{\pi}{2}\right]$ :

$$
\begin{gathered}
S^{0}(-\varphi)=-S^{0}(\varphi), \quad U^{0}(-\varphi)=-U^{0}(\varphi), \quad Z_{b}^{0}(-\varphi)=-Z_{b}^{0}(\varphi) \\
0<\varphi \leq \frac{\pi}{2} \Rightarrow S^{0}(\varphi)=S(\varphi), U^{0}(\varphi)=U(\varphi), Z_{b}^{0}(\varphi)=Z_{b}(\varphi)
\end{gathered}
$$

Then, the description 65 of the stable and unstable manifolds of $(0,0)$ may be replaced by

$$
\begin{aligned}
& \mathcal{S}^{0}=\left\{(\psi, \varphi) \in \mathcal{C}, \varphi \neq 0 \text { and } \psi=S^{0}(\varphi)\right\} \cup[-\bar{\sigma}, \bar{\sigma}] \times\{0\} \\
& \mathcal{U}^{0}=\left\{(\psi, \varphi) \in \mathcal{C}, \psi=U^{0}(\varphi)\right\}
\end{aligned}
$$

and 58 may be replaced by

$$
b(\psi, \varphi)=0 \Leftrightarrow\left\{\begin{array}{l}
\varphi=0 \text { and } \psi \in[-\bar{\sigma}, \bar{\sigma}] \cup[\pi-\bar{\sigma}, \pi+\bar{\sigma}] \\
\text { or } \quad \varphi \neq 0 \text { and } \psi \in\left\{Z_{b}^{0}(\varphi)\right\} \cup\left\{Z_{b}^{\pi}(\varphi)\right\}
\end{array}\right.
$$

The equilibrium point $(\pi, 0)$. The " + " symmetry (see $(81)$ ) obviously maps $(0,0)$ to $(\pi, 0)$, the stable manifold of $(0,0)$ to the unstable manifold of $(\pi, 0)$, the unstable one to the stable one and the set of zeroes of $b$ to itself. Define $\mathcal{S}^{\pi}, \mathcal{U}^{\pi}, Z_{b}^{\pi}$ after $\mathcal{S}^{0}, \mathcal{U}^{0}, Z_{b}^{0}$ by

$$
S^{\pi}(\varphi)=\pi+U^{0}(\varphi), \quad U^{\pi}(\varphi)=\pi+S^{0}(\varphi), \quad Z_{b}^{\pi}(\varphi)=\pi+Z_{b}^{0}(\varphi)
$$

From 84, , 85) and $(87)$, the relation $(66)$ translates into

$$
\begin{array}{r}
0<\varphi<\frac{\pi}{2} \Rightarrow\left\{\begin{array}{l}
S^{0}(\varphi)<Z_{b}^{0}(\varphi)<0<U^{0}(\varphi), \\
U^{\pi}(\varphi)<Z_{b}^{\pi}(\varphi)<\pi<S^{\pi}(\varphi),
\end{array}\right. \\
-\frac{\pi}{2}<\varphi<0 \Rightarrow\left\{\begin{array}{l}
U^{0}(\varphi)<0<Z_{b}^{0}(\varphi)<S^{0}(\varphi), \\
S^{\pi}(\varphi)<\pi<Z_{b}^{\pi}(\varphi)<U^{\pi}(\varphi) .
\end{array}\right.
\end{array}
$$

The stable and unstable manifolds of $(\pi, 0)$ are:

$$
\begin{aligned}
& \mathcal{S}^{\pi}=\left\{(\psi, \varphi) \in \mathcal{C}, \psi=S^{\pi}(\varphi)\right\}, \\
& \mathcal{U}^{\pi}=\left\{(\psi, \varphi) \in \mathcal{C}, \varphi \neq 0 \text { and } \psi=U^{\pi}(\varphi)\right\} \cup[-\bar{\sigma}, \bar{\sigma}] \times\{0\} .
\end{aligned}
$$

Also, 58 and 59 become:

$$
\begin{gathered}
b(\psi, \varphi)=0 \Leftrightarrow\left\{\begin{array}{c}
\varphi=0 \text { and } \psi \in[-\bar{\sigma}, \bar{\sigma}] \cup[\pi-\bar{\sigma}, \pi+\bar{\sigma}] \\
\text { or } \quad \varphi \neq 0 \text { and } \psi \in\left\{Z_{b}^{0}(\varphi)\right\} \cup\left\{Z_{b}^{\pi}(\varphi)\right\}
\end{array}\right. \\
b(\psi, \varphi)>0 \text { if }\left\{\begin{array}{c}
\varphi=0 \text { and } \bar{\sigma}<\psi<\pi+\bar{\sigma} \\
\text { or } \quad \varphi \neq 0 \text { and } Z_{b}^{0}(\varphi)<\psi<Z_{b}^{\pi}(\varphi) .
\end{array}\right.
\end{gathered}
$$




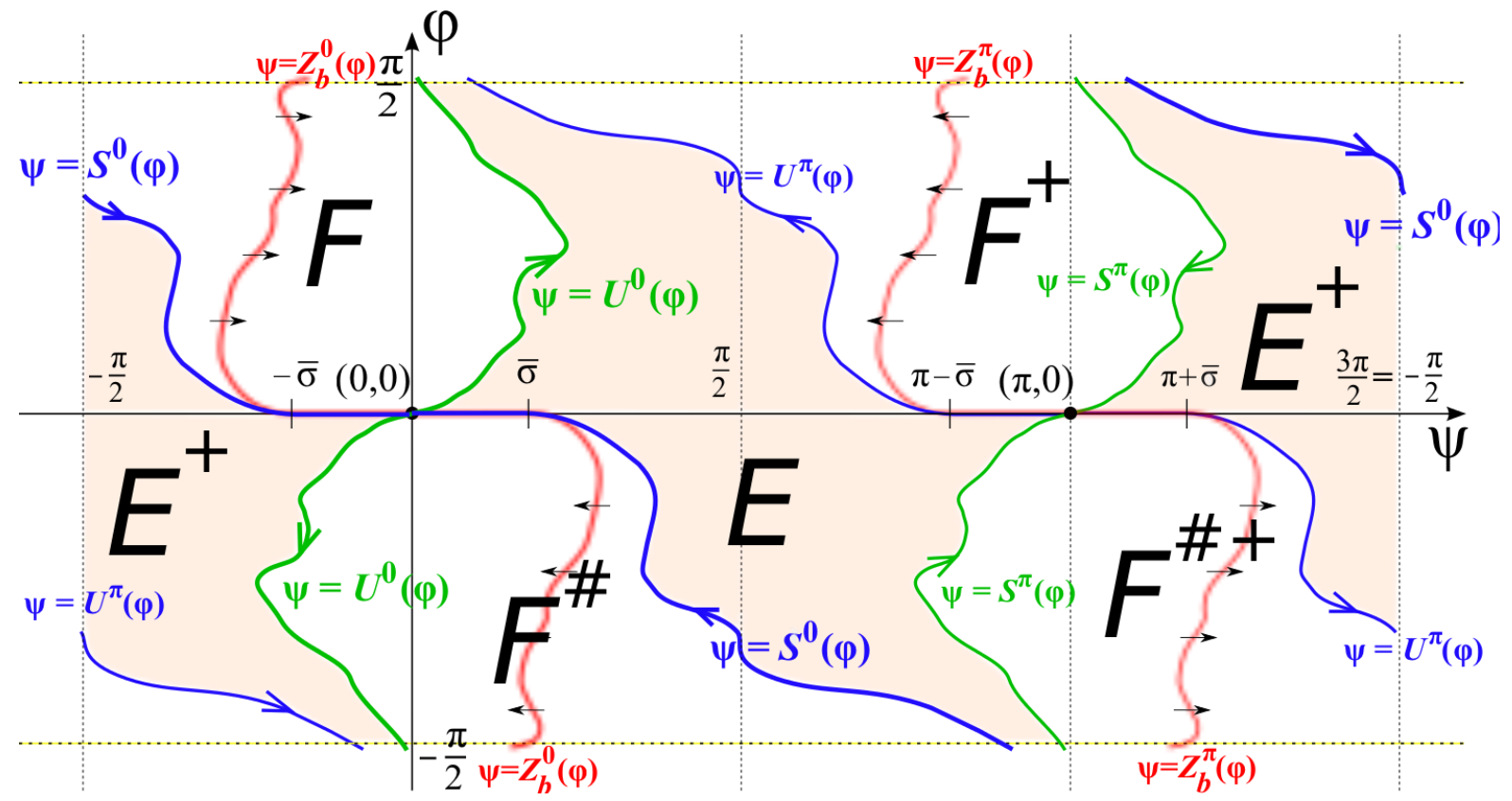

Fig. 7 This picture reflects qualitatively the assumptions on $a$ and $b$. It is provided as a help to follow the proof; a precise numerical drawing is provided in Figure 1 and Figure 2 for the specific expression of $a, b$ in the case of full control or tangential thrust.

The six invariant regions separated by the stable and unstable manifolds of $(0,0)$ and $(\pi, 0)$ are shown. The other curves are $\psi=Z_{b}^{0}(\varphi)$ and $\psi=Z_{b}^{\pi}(\varphi)$, where $b(\psi, \varphi)$ changes sign.

Invariant regions of $\mathcal{C}$. The stable and unstable manifolds $\mathcal{S}^{0}, \mathcal{U}^{0}, \mathcal{S}^{\pi}, \mathcal{U}^{\pi}$, that intersect at the equilibria $(0,0)$ and $(\pi, 0)$ are invariant sets that divide the cylinder $\mathcal{C}$ into six open regions:

$$
\begin{aligned}
F & =\left\{(\psi, \varphi), 0<\varphi<\pi / 2 \text { and } S^{0}(\varphi)<\psi<U^{0}(\varphi)\right\}, \\
F^{+} & =\left\{(\psi, \varphi), 0<\varphi<\pi / 2 \text { and } U^{\pi}(\varphi)<\psi<S^{\pi}(\varphi)\right\}, \\
F^{\sharp} & =\left\{(\psi, \varphi),-\pi / 2<\varphi<0 \text { and } U^{0}(\varphi)<\psi<S^{0}(\varphi)\right\}, \\
F^{\sharp+} & =\left\{(\psi, \varphi),-\pi / 2<\varphi<0 \text { and } S^{\pi}(\varphi)<\psi<U^{\pi}(\varphi)\right\}, \\
E & =\left\{(\psi, \varphi), S^{0}(\varphi)<\psi<S^{\pi}(\varphi) \text { if } \varphi \leq 0, U^{0}(\varphi)<\psi<U^{\pi}(\varphi) \text { if } \varphi \geq 0\right\}, \\
E^{+} & =\left\{(\psi, \varphi), U^{\pi}(\varphi)<\psi<U^{0}(\varphi) \text { if } \varphi \leq 0, S^{\pi}(\varphi)<\psi<S^{0}(\varphi) \text { if } \varphi \geq 0\right\} .
\end{aligned}
$$

$E$ is self-symmetric under the " $\sharp$ " symmetry and $E^{+}$is its own image under the "+" symmetry; $F^{+}, F^{\sharp}, F^{\sharp+}$ are the images of $F$ by the " $\sharp$ " and "+" symmetries; see Lemma 1 These regions are represented in Figure 7.

We now state and prove two preliminary lemmas and give the proper proof of Theorem 3 .

Lemma 2 Assume that a, $b$ satisfy assumptions (53) to (60), and consider a solution $t \mapsto(\psi(t), \varphi(t))$ of (54) defined on $\left[0, \tau_{\text {fin }}\right], \tau_{\text {fin }}>0$.

1. If it starts in $E$ or in the upper part of the unstable manifold $\mathcal{U}^{0}, \varphi($.$) is monotonic increasing.$

2. If it starts in $F$, then

- if it starts in $\left\{(\psi, \varphi), Z_{b}^{0}(\varphi) \leq \psi<U^{0}(\varphi)\right\}$, it remains in this part of $E$ and $\varphi($.$) is monotonic increasing,$

- if it starts in $\left\{(\psi, \varphi), S^{0}(\varphi)<\psi<Z_{b}^{0}(\varphi)\right\}$, either it remains in this part of E and $\varphi($.$) is monotonic decreasing,$ or there is some $\bar{\tau}, 0<\bar{\tau}<\tau_{\text {fin }}$ such that $t \mapsto \varphi(t)$ is monotonic decreasing for $t$ between 0 and $\bar{\tau}$, minimum for $t=\bar{\tau}$ and monotonic increasing for $t$ between $\bar{\tau}$ and $\tau_{\text {fin }}$, with $\psi(\bar{\tau})-Z_{b}^{0}(\varphi(\bar{\tau}))=b(\psi(\bar{\tau}), \varphi(\bar{\tau}))=0$.

3. If it starts in the upper part of the stable manifold $\mathcal{S}^{0}, \varphi($.$) is monotonic non-increasing.$

The behavior in the regions $E^{+}, F^{+}, F^{\sharp}, F^{\sharp+}$ and on the other pieces of stable or unstable curves are obtained by symmetry; see Lemma 1 .

Proof Points 1 and 3 are obvious because $b$ is negative in $E$ and in the upper part of $\mathcal{U}^{0}$ while it is positive in the upper part of $\mathcal{S}^{0}$ except, if $\bar{\sigma}$ is nonzero, on the segment $\{\varphi=0\}$, where it is zero. Let us prove point 2 . In the region $F$, according to $59,, b$ has the sign of $\psi-Z_{b}^{0}(\varphi)$. Using differentiability of $Z_{b}^{0}$ away from $\varphi=0$ (see (63)), 
one may compute the derivative of $\psi-Z_{b}^{0}(\varphi)$ with respect to time along a solution; it is is $a(\psi, \varphi)-Z_{b}^{0^{\prime}}(\varphi) b(\psi, \varphi)$, which, according to 600 , is positive when $\psi-Z_{b}^{0}(\varphi)=0$, i.e. when $b(\psi, \varphi)=0$. Hence the region where $b>0$ is positively invariant, this accounts for the behavior of solutions starting in $\{b>0\}$, and no solution may stay on the locus where $b=0$, this accounts for solutions that start in $\{b<0\}$ : either they stay in this part of $F$ or they cross $\{b=0\}$ at one time and then remain in $\{b>0\}$.

For any number $f, 0<f<\frac{\pi}{2}$, let

$$
\mathcal{C}_{f}=\{(\psi, \varphi) \in \mathcal{C},|\varphi|<f\} .
$$

Lemma 3 Assume that a, b, c satisfy assumptions (53) to 62. For any number $f, 0<f<\frac{\pi}{2}$, there are two neighborhoods $\Omega_{0}$ and $\Omega_{\pi}$ of $(0,0)$ and $(\pi, 0)$ respectively and, a number $T(f) \geq 0$ such that

$$
\begin{aligned}
(\psi, \varphi) \in \Omega_{0} & \Rightarrow c(\psi, \varphi)>\frac{1}{2}, \quad(\psi, \varphi) \in \Omega_{\pi} \Rightarrow c(\psi, \varphi)<-\frac{1}{2}, \\
\text { no solution } t & \mapsto(\psi(t), \varphi(t)) \text { of } 54 \text { may cross both } \Omega_{0} \text { and } \Omega_{\pi},
\end{aligned}
$$

and any solution $t \mapsto(\psi(t), \varphi(t))$ defined on the time interval $\left[0, \tau_{\text {fin }}\right]$ such that $(\psi(t), \varphi(t)) \in \mathcal{C}_{f}$ for all $t \in$ $\left[0, \tau_{\text {fin }}\right]$ satisfies

$$
\text { meas }\left\{t \in\left[0, \tau_{\text {fin }}\right],(\psi(t), \varphi(t)) \notin\left(\Omega_{0} \cup \Omega_{\pi}\right)\right\} \leq T(f) .
$$

The left-hand side (meas stands for the Lebesgue measure of a subset of $\mathbb{R}$ ) is simply the time spent by the solution outside the neighborhoods $\Omega_{0}$ and $\Omega_{\pi}$ of $(0,0)$ and $(\pi, 0)$; this bound depends on $f<\frac{\pi}{2}$ because $a$ and $b$ could tend to zero as $\varphi$ tends to $\pm \frac{\pi}{2}$.

Proof of Lemma 3 To deal with the case where the stable or unstable manifolds contain a segment of the $\psi$-axis, we must account for both the cases where $\bar{\sigma}=0$ and where $\bar{\sigma} \neq 0$. We define the set $\mathcal{K}^{\varepsilon}(\varepsilon>0)$ as follows:

$$
\mathcal{K}^{\varepsilon}=\varnothing \text { if } \bar{\sigma}=0
$$

and, if $\bar{\sigma} \neq 0$,

$$
\begin{aligned}
\mathcal{K}^{\varepsilon}= & {[\varepsilon, \bar{\sigma}+\varepsilon] \times[-\bar{k} \varepsilon, \bar{k} \varepsilon] \cup[-\bar{\sigma}-\varepsilon,-\varepsilon] \times[-\bar{k} \varepsilon, \bar{k} \varepsilon] } \\
& \cup[\pi+\varepsilon, \pi+\bar{\sigma}+\varepsilon] \times[-\bar{k} \varepsilon, \bar{k} \varepsilon] \cup[\pi-\bar{\sigma}-\varepsilon, \pi-\varepsilon] \times[-\bar{k} \varepsilon, \bar{k} \varepsilon]
\end{aligned}
$$

with

$$
0<\bar{k} \leq \min \left\{1, \frac{1}{2}\left|\frac{\partial a}{\partial \psi}(0,0) / \frac{\partial a}{\partial \varphi}(0,0)\right|\right\} .
$$

Since $\frac{\partial b}{\partial \psi}(0,0)$ is zero, 61], (59) and (60) imply $\frac{\partial b}{\partial \varphi}(0,0)>0, \frac{\partial a}{\partial \psi}(0,0)<0$, and 66) implies $\frac{\partial a}{\partial \varphi}(0,0) \geq 0$. The slope of the tangent to the curve $a=0$ is $-\frac{\partial a}{\partial \psi}(0,0) / \frac{\partial a}{\partial \varphi}(0,0)$, and the slope of the unstable manifold $\left\{\psi=U^{0}(\varphi)\right\}$ (i.e. the slope of the eigenvector corresponding to the negative eigenvalue $\frac{\partial a}{\partial \psi}(0,0)$ ) is $\left(\partial b / \partial \varphi(0,0)-\frac{\partial a}{\partial \psi}(0,0)\right) / \frac{\partial a}{\partial \varphi}(0,0)$, larger than the previous slope. Hence, for some open ball $B$ around the origin,

$$
|\varphi| \leq \bar{k}|\psi|, \psi \neq 0,(\psi, \varphi) \in B \Rightarrow|a(\psi, \varphi)| \neq 0 \text { and }\left|\psi-U^{0}(\varphi)\right| \neq 0 .
$$

This implies that $a$ does not vanish on $B \cap([\varepsilon, \bar{\sigma}+\varepsilon] \times[-\bar{k} \varepsilon, \bar{k} \varepsilon])$. On the compact segment $\{(\psi, 0), 0 \leq \psi \leq$ $\bar{\sigma},(\psi, 0) \notin B\},|a(\psi, \varphi)|$ has a positive lower bound $\underline{a}$; hence, for $\varepsilon$ small enough, $a(\psi, \varphi)$ is larger that $\frac{1}{2} \underline{a} / 2$ on the part of the compact rectangle $[\varepsilon, \bar{\sigma}+\varepsilon] \times[-\bar{k} \varepsilon, \bar{k} \varepsilon]$ that is outside $B$. Gluing the piece inside $B$ and the piece outside $B$ together, we get that, for $\varepsilon$ small enough, $a$ does not vanish on the compact rectangle $[\varepsilon, \bar{\sigma}+\varepsilon] \times$ $[-\bar{k} \varepsilon, \bar{k} \varepsilon]$. By symmetry, i.e. from (53) and (103), we get that, for $\varepsilon$ small enough,

$$
a \text { does not vanish on } \mathcal{K}^{\varepsilon} \text {. }
$$

By a similar argument, since $\psi-U^{0}(\varphi)$ does not vanish on the compact segment $\{(\psi, 0), 0 \leq \psi \leq \bar{\sigma},(\psi, 0) \notin$ $B\}$, (105) implies that it also does not vanish on $\mathcal{K}^{\varepsilon}$ for $\varepsilon$ small enough either, hence

$$
\mathcal{U}^{0} \cap \mathcal{K}^{\varepsilon}=\mathcal{U}^{\pi} \cap \mathcal{K}^{\varepsilon}=\varnothing
$$

for $\varepsilon$ small enough. Define the neighborhoods $\Omega_{0}^{\varepsilon}$ and $\Omega_{\pi}^{\varepsilon}$ of $(0,0)$ and $(\pi, 0)$ as:

$$
\begin{aligned}
\Omega_{0}^{\varepsilon} & =\{(\psi, \varphi),|\psi|<\varepsilon,|\varphi|<\varepsilon\}, \\
\Omega_{\pi}^{\varepsilon}=\left(\Omega_{0}^{\varepsilon}\right)^{+} & =\{(\psi, \varphi),|\psi-\pi|<\varepsilon,|\varphi|<\varepsilon\} .
\end{aligned}
$$


Consider the two distinct solutions going through $\left(\frac{\pi}{2}, 0\right)$ and $\left(-\frac{\pi}{2}, 0\right)$; for $\varepsilon$ small enough they cross neither $\Omega_{\pi}^{\varepsilon}$ nor $\Omega_{\pi}^{0}$, and hence they separate $\mathcal{C}$ into two regions, one containing $\Omega_{\pi}^{\varepsilon}$ and the other one $\Omega_{\pi}^{\varepsilon}$. Hence, for $\varepsilon$ small enough, no solution can cross both $\Omega_{\pi}^{\varepsilon}$ and $\Omega_{0}^{\varepsilon}$. From (62), it is also clear that, for $\varepsilon$ small enough,

$$
\min _{(\psi, \varphi) \in \Omega_{0}^{\varepsilon}} c(\psi, \varphi)>\frac{1}{2} \text { and } \max _{(\psi, \varphi) \in \Omega_{\pi}^{\varepsilon}} c(\psi, \varphi)<-\frac{1}{2}
$$

Let us now fix some $\varepsilon$ small enough that this is true, 106 and 107 hold and no solution can cross both $\Omega_{\pi}^{\varepsilon}$ and $\Omega_{0}^{\varepsilon}$. Take

$$
\Omega_{0}=\Omega_{0}^{\varepsilon}, \Omega_{\pi}=\Omega_{\pi}^{\varepsilon}, \mathcal{K}=\mathcal{K}^{\varepsilon}
$$

for this fixed value of $\varepsilon$. With this choice, one has

$$
\mathcal{U}^{0} \cap \mathcal{K}=\mathcal{U}^{\pi} \cap \mathcal{K}=\varnothing
$$

and there is some $\underline{a}>0$ such that

$$
(\psi, \varphi) \in \mathcal{K} \Rightarrow|a(\psi, \varphi)|>\underline{a}
$$

and $(99)$ and $(100)$ hold: we only need to prove that (101) holds as well.

First, "thicken" the curves $\psi=Z_{b}^{0}(\varphi)$ and $\psi=Z_{b}^{\pi}(\varphi)$ where $b$ vanishes, using the flow $\Phi$ (see (55)):

$$
\begin{aligned}
\Sigma_{0} & =\left\{\Phi\left(Z_{b}^{0}(\varphi), \varphi, t\right),-\frac{\pi}{2}<\varphi<\frac{\pi}{2}, \varphi \neq 0,-\frac{1}{2}<t<\frac{1}{2}\right\} \cap \mathcal{C}_{f}, \\
\Sigma_{\pi}=\left(\Sigma_{0}\right)^{+} & =\left\{\Phi\left(Z_{b}^{\pi}(\varphi), \varphi, t\right),-\frac{\pi}{2}<\varphi<\frac{\pi}{2}, \varphi \neq 0,-\frac{1}{2}<t<\frac{1}{2}\right\} \cap \mathcal{C}_{f} .
\end{aligned}
$$

Note: if, for the initial condition $\left(\psi^{o}, \varphi^{o}\right)=\left(Z_{b}^{\pi}(\varphi), \varphi\right)$, either $\tau^{-}>-\frac{1}{2}$ or $\tau^{+}<\frac{1}{2}$ (see (54)-(61)), then $\Phi\left(Z_{b}^{\pi}(\varphi), \varphi, t\right)$ is not defined up to $-\frac{1}{2}$ or $\frac{1}{2}$; we however kept, for the sake of simplicity, "- $\frac{1}{2}<t<\frac{1}{2}$ " instead of " $\max \left\{-\frac{1}{2}, \tau^{-}\right\}<t<\min \left\{\frac{1}{2}, \tau^{+}\right\}$".

The topological closure of $\mathcal{C}_{f} \backslash\left(\Omega_{0} \cup \Omega_{\pi} \cup \mathcal{K} \cup \Sigma_{0} \cup \Sigma_{\pi}\right)$ does not contain any zero of $b$, and is obviously compact. Hence $b$ has a positive lower bound $m(f)$ on that compact set:

$$
(\psi, \varphi) \in \mathcal{C}_{f} \backslash\left(\Omega_{0} \cup \Omega_{\pi} \cup \mathcal{K} \cup \Sigma_{0} \cup \Sigma_{\pi}\right) \Rightarrow|b(\psi, \varphi)|>m(f)>0 .
$$

Now consider a solution $\left[0, \tau_{\text {fin }}\right] \rightarrow \mathcal{C}_{f}$, and partition $\left[0, \tau_{\text {fin }}\right]$ as follows:

$$
\begin{aligned}
{\left[0, \tau_{\text {fin }}\right]=I_{0} \cup I_{1} \cup I_{2} \cup I_{3} } \\
\text { with } I_{0}=\left\{t \in\left[0, \tau_{\text {fin }}\right],(\psi(t), \varphi(t)) \in \Omega_{0} \cup \Omega_{\pi}\right\}, \\
I_{1}=\left\{t \in\left[0, \tau_{\text {fin }}\right],(\psi(t), \varphi(t)) \in \mathcal{K}\right\}, \\
I_{2}=\left\{t \in\left[0, \tau_{\text {fin }}\right],(\psi(t), \varphi(t)) \in \Sigma_{0} \cup \Sigma_{\pi} \text { and } t \notin I_{0} \cup I_{1}\right\}, \\
I_{3}=\left\{t \in\left[0, \tau_{\text {fin }}\right],(\psi(t), \varphi(t)) \notin \Omega_{0} \cup \Omega_{\pi} \cup \mathcal{K} \cup \Sigma_{0} \cup \Sigma_{\pi}\right\} .
\end{aligned}
$$

Obviously,

$$
\text { meas }\left\{t \in\left[0, \tau_{\text {fin }}\right],(\psi(t), \varphi(t)) \notin\left(\Omega_{0} \cup \Omega_{\pi}\right)\right\}=\text { meas } I_{1}+\text { meas } I_{2}+\text { meas } I_{3} \text {. }
$$

Either the solution is one of the two equilibria or it stays in one of the stable or unstable manifolds or in one of the six regions $E, E^{+}, F, F^{+}, F^{\sharp}, F^{\sharp+}$. According to Lemma 1 , and seen that the neighborhoods are invariant by the $\sharp$ symmetry and exchanged by the + symmetry, it is enough to prove the property for solutions in the regions $E$ and $F$, the equilibrium $(0,0)$ and the upper parts of its stable and unstable manifolds $\mathcal{S}^{0}$ and $\mathcal{U}^{0}$.

In order to bound meas $I_{1}$ if $\bar{\sigma} \neq 0$ (it is zero if $\bar{\sigma}=0$ ), let us prove that

either the solution does not cross $\mathcal{K}$

or there are times $t_{1}, t_{2}, 0 \leq t_{1}<t_{2} \leq \tau_{\text {fin }}$ such that the solution is

in $\mathcal{K}$ on the time interval $\left[t_{1}, t_{2}\right]$ and outside $\mathcal{K}$ on $\left[0, \tau_{\text {fin }}\right] \backslash\left[t_{1}, t_{2}\right]$.

- This is obvious if the solution is an equilibrium or is on the unstable manifold $\mathcal{U}^{0}$, that do not cross $\mathcal{K}$ (see (110). 
- In $E$ : among the rectangles in (103), only $[\varepsilon, \bar{\sigma}+\varepsilon] \times[-\bar{k} \varepsilon, \bar{k} \varepsilon]$ and $[\pi-\bar{\sigma}-\varepsilon, \pi-\varepsilon] \times[-\bar{k} \varepsilon, \bar{k} \varepsilon]$ intersect $E$; a solution that lies in $E$ cannot cross both rectangles because the solution passing through $\left(0, \frac{\pi}{2}\right)$ separates them. Consider a solution that crosses one of them, say $[\varepsilon, \bar{\sigma}+\varepsilon] \times[-\bar{k} \varepsilon, \bar{k} \varepsilon]$ (the situation around $[\pi-\bar{\sigma}-$ $\varepsilon, \pi-\varepsilon] \times[-\bar{k} \varepsilon, \bar{k} \varepsilon]$ is similar). Since $\dot{\psi}$ is negative in the rectangle ( $a$ does not change sign according to (106) and $a(0, \bar{\sigma})<0$ according to (60) $)$ and $\dot{\varphi}$ is positive in $E$, a solution may only exit through the top or left-hand edge; if it exits through the top edge $\{\varphi=\bar{k} \varepsilon\}$, it will not enter again because $\varphi$ will remain larger than $\bar{k} \varepsilon$. If it exits through the left-hand edge, the fact that $\dot{\varphi}>0$ in $E$ only allows it to enter again through the same edge, but this is impossible because $\dot{\psi}>0$ on this edge; this proves (116).

- In $F$ : from (110), $[-\bar{\sigma}-\varepsilon,-\varepsilon] \times[-\bar{k} \varepsilon, \bar{k} \varepsilon]$ is the only rectangle in $[103)$ that intersects $F$; hence the solution may only cross this rectangle. Since, according to $106, a$ does not change sign in the rectangle and, according to (60), $a(0, \bar{\sigma})>0, a$ is positive on the rectangle, then the vector field points inwards on the left-hand edge $\{\psi=-\bar{\sigma}-\varepsilon\}$ and outwards on the right-hand edge $\{\psi=-\varepsilon\}$. The bottom edge is not in $F$. A solution may only exit through the top or right-hand edge; if it exits through the top edge, it means that $\varphi(t)$ is increasing at the exit time, and so, according to Lemma 2 , it will continue increasing and cannot go back to the rectangle. Also, if it exits through the right-hand edge, re-entering the rectangle through the top or left-hand edge would require $\varphi$ to increase and reach at least $k \varepsilon$, making it impossible to reach the rectangle again because $\varphi$ will continue increasing. This proves $(116)$ for solutions that remain in $F$.

- A solution in the upper part of the stable manifold $\mathcal{S}^{0}$ also satisfies $(116)$ because it enters the rectangle through the left-hand edge or the top edge and exits it through the right-hand edge and then goes asymptotically to $(0,0)$.

We have proved (116) for any solution. Either $I_{1}=\varnothing$ or $I_{1}=\left[t_{1}, t_{2}\right]$ and connectedness implies that the solution stays in a single rectangle of $\mathcal{K}$. Using [111], $\psi(t)$ varies monotonically in $\left[t_{1}, t_{2}\right]$, and so its variation is at most $\bar{\sigma}: \underline{a}\left(t_{2}-t_{1}\right)<\bar{\sigma}$. This yields

$$
\text { meas } I_{1} \leq \bar{\sigma} / \underline{a} .
$$

Solutions in $E$ or in the stable and unstable manifolds cross neither $\Sigma_{0}$ nor $\Sigma_{\pi}$; hence $I_{2}=\varnothing$ for these solutions. Solutions in $F$ may cross $\Sigma_{0}$ but not $\Sigma_{\pi}$, and they cannot enter $\Sigma_{0}$ again after leaving it because the region between $\Sigma_{0}$ and the unstable manifold $\mathcal{U}^{0}$ is invariant in positive time. They stay in $\Sigma_{0}$ on a time-interval of length at most 1 from the definition 112 . Hence, for any solution,

$$
\text { meas } I_{2} \leq 1 \text {. }
$$

Using Lemma 2, for any solutions in $E$ or $F$ or the upper part of $\mathcal{S}^{0}$ or $\mathcal{U}^{0}$, the total variation of $\varphi$ on the interval $\left[0, \tau_{\text {fin }}\right]$ is at most $\pi-2 \ell$. The inequality (113) then implies

$$
\text { meas } I_{3} \leq(\pi-2 \ell) / m(f) \text {. }
$$

Setting $T(f)=\bar{\sigma} / \underline{a}+1+(\pi-2 \ell) / m(f)$, (115), (117), (118) and (119) imply (101).

Proof of Theorem 3 From Lemma 1, the "+" symmetry allows one to interchange $\varphi^{1}$ and $\varphi^{0}$ while the "\#” symmetry changes their sign: we may assume

$$
-\frac{\pi}{2}<\varphi^{0} \leq \varphi^{1}<\frac{\pi}{2} \text { and } \varphi^{1} \geq 0
$$

in the proof without loss of generality. We distinguish four cases.

Case a: $-\frac{\pi}{2}<\varphi^{0} \leq 0<\varphi^{1}<\frac{\pi}{2}$. In this paragraph, by convention,

$$
\text { if } \varphi^{0}=0 \text {, then } S^{0}\left(\varphi^{0}\right) \text { stands for } \bar{\sigma} \text { and } S^{\pi}\left(\varphi^{0}\right) \text { stands for } \pi-\bar{\sigma} \text {. }
$$

The solutions such that $\varphi(0)=\varphi^{0}, \varphi\left(\tau_{\text {fin }}\right)=\varphi^{1}$, must be in the region $E$ (see Lemma 2 and Figure 7 ), and satisfy $S^{0}\left(\varphi^{0}\right)<\psi(0)<S^{\pi}\left(\varphi^{0}\right)$. For any $\chi$ in the open interval $\left(S^{0}\left(\varphi^{0}\right), S^{\pi}\left(\varphi^{0}\right)\right)$, let $\left(\psi^{\chi}(),. \varphi^{\chi}().\right)$ be the unique solution to the Cauchy problem (54) with initial condition

$$
\psi^{\chi}(0)=\chi, \varphi^{\chi}(0)=\varphi^{0} .
$$

It is - see [55] - continuous with respect to $\chi$ and continuously differentiable with respect to $\tau$. Since this solution is in $E, \varphi^{\chi}$ is an increasing function of time, and so there is a unique time $\tau_{\text {fin }}^{\chi}>0$ such that

$$
\varphi^{\chi}\left(\tau_{\text {fin }}^{\chi}\right)=\varphi^{1} .
$$


Since $b\left(\varphi^{\chi}\left(\tau_{\text {fin }}^{\chi}\right), \psi^{\chi}\left(\tau_{\text {fin }}^{\chi}\right)\right)>0$, there is a constant $k>0$ such that $\left|\varphi^{\chi}\left(t^{\prime}\right)-\varphi^{\chi}\left(t^{\prime \prime}\right)\right|>k\left|t^{\prime}-t^{\prime \prime}\right|$ for $t^{\prime}, t^{\prime \prime}$ in a neighborhood of $\tau_{\text {fin }}^{\chi}$. This implies that $\tau_{\text {fin }}^{\chi}$ depends continuously on $\chi$. This allows us to define a continuous map $\Lambda:\left(S^{0}\left(\varphi^{0}\right), S^{\pi}\left(\varphi^{0}\right)\right) \rightarrow \mathbb{R}$ by

$$
\Lambda(\chi)=\int_{0}^{\tau_{\text {fin }}^{\chi}} c\left(\psi^{\chi}(s), \varphi^{\chi}(s)\right) \mathrm{d} s .
$$

All we need to prove is that, for any $\bar{\lambda} \in \mathbb{R}$, there is at least one $\chi$ in $\left(S^{0}\left(\varphi^{0}\right), S^{\pi}\left(\varphi^{0}\right)\right)$ such that $\Lambda(\chi)=\bar{\lambda}$, i.e. that $\Lambda$ is onto. Since $\Lambda$ is continuous, it is sufficient to prove that

$$
\lim _{\substack{\chi \rightarrow S^{0}\left(\varphi^{0}\right) \\ \chi>S^{0}\left(\varphi^{0}\right)}} \Lambda(\chi)=+\infty, \lim _{\substack{\chi \rightarrow S^{\pi}\left(\varphi^{0}\right) \\ \chi<S^{\pi}\left(\varphi^{0}\right)}} \Lambda(\chi)=-\infty .
$$

The solution $t \mapsto\left(\psi^{S^{0}\left(\varphi^{0}\right)}(t), \varphi^{S^{0}\left(\varphi^{0}\right)}(t)\right)$ of 54 with initial condition $\left(S^{0}\left(\varphi^{0}\right), \varphi^{0}\right)$ is on the stable manifold of $(0,0)$; it is defined on $[0,+\infty) ; \varphi^{S^{0}\left(\varphi^{0}\right)}(t)$ is negative for all time and tends to zero as $t \rightarrow+\infty$. By continuity with respect to initial conditions, the solutions $\left(\psi^{\chi}(),. \varphi^{\chi}().\right)$ starting from $\left(\chi, \varphi^{0}\right)$ with $\chi$ close enough to $S^{0}\left(\varphi^{0}\right)$ are also defined on $[0, \bar{\tau}]$ for arbitrarily large fixed $\bar{\tau}>0$, and converge uniformly to $\left(\psi^{S^{0}\left(\varphi^{0}\right)}(),. \varphi^{S^{0}\left(\varphi^{0}\right)}().\right)$ on the compact interval $[0, \bar{\tau}]$ as $\chi \rightarrow S^{0}\left(\varphi^{0}\right)$. This proves that, for $\chi$ close enough to $S^{0}\left(\varphi^{0}\right), \varphi^{\chi}(\bar{\tau})<0$ and hence $\tau_{\text {fin }}>\bar{\tau}$. The situation near $S^{\pi}\left(\varphi^{0}\right)$ being similar, we have proved that

$$
\lim _{\substack{\chi \rightarrow S^{0}\left(\varphi^{0}\right) \\ \chi>S^{0}\left(\varphi^{0}\right)}} \tau_{\text {fin }}^{\chi}=\lim _{\substack{\chi \rightarrow S^{\pi}\left(\varphi^{0}\right) \\ \chi<S^{\pi}\left(\varphi^{0}\right)}} \tau_{\text {fin }}^{\chi}=+\infty .
$$

Now define $\Omega_{0}$ and $\Omega_{\pi}$ according to Lemma 3 , and $\bar{\tau}$ large enough that $\left(\psi^{S^{0}\left(\varphi^{0}\right)}(\bar{\tau}), \varphi^{S^{0}\left(\varphi^{0}\right)}(\bar{\tau})\right)$ is in $\Omega_{0}$. For $\chi$ close enough to $S^{0}\left(\varphi^{0}\right)$, we also have $\left(\psi^{\chi}(\bar{\tau}), \varphi^{\chi}(\bar{\tau})\right) \in S^{0}\left(\varphi^{0}\right)$; hence, according to (100), solutions $\left(\psi^{\chi}(),. \varphi^{\chi}().\right)$ with $\chi$ close enough to $S^{0}\left(\varphi^{0}\right)$ never cross $\Omega_{\pi}$. The interval $\left[0, \tau_{\text {fin }}^{\chi}\right]$ is partitioned into times $t$ such that $\left(\psi^{\chi}(t), \varphi^{\chi}(t)\right) \in \Omega_{0}$ and also $\left(\psi^{\chi}(t), \varphi^{\chi}(t)\right) \notin\left(\Omega_{0} \cup \Omega_{\pi}\right)$. Since, according to 101) ( with $f=$ $\left.\max \left\{\left|\varphi^{0}\right|,\left|\varphi^{1}\right|\right\}\right)$ meas $\left\{t \in\left[0, \tau_{\text {fin }}^{\chi}\right],\left(\psi^{\chi}(t), \varphi^{\chi}(t)\right) \notin\left(\Omega_{0} \cup \Omega_{\pi}\right)\right\} \leq T(f)$ and meas $\left\{t \in\left[0, \tau_{\text {fin }}^{\chi}\right],\left(\psi^{\chi}(t), \varphi^{\chi}(t)\right) \in\right.$ $\left.\Omega_{0}\right\} \geq \tau_{\text {fin }}^{\chi}-T(f)$, then (124) and 99 imply

$$
\Lambda(\chi) \geq \frac{1}{2}\left(\tau_{\text {fin }}^{\chi}-T(f)\right)-c_{f} T(f) \quad \text { with } \quad c_{f}=\max _{(\psi, \varphi) \in \mathcal{C},|\varphi| \leq f}|c(\psi, \varphi)|,
$$

and this does imply, using (126), the first limit in 125). Similarly, for $\chi$ close enough to $S^{\pi}\left(\varphi^{0}\right)$, one has meas $\{t \in$ $\left.\left[0, \tau_{\text {fin }}^{\chi}\right],\left(\psi^{\chi}(t), \varphi^{\chi}(t)\right) \in \Omega_{\pi}\right\} \geq \tau_{\text {fin }}^{\chi}-T(f)$, meas $\left\{t \in\left[0, \tau_{\text {fin }}^{\chi}\right],\left(\psi^{\chi}(t), \varphi^{\chi}(t)\right) \notin\left(\Omega_{0} \cup \Omega_{\pi}\right)\right\} \leq T(f)$ and hence, using (127) again,

$$
\Lambda(\chi) \leq-\frac{1}{2}\left(\tau_{\text {fin }}^{\chi}-T(f)\right)+c_{f} T(f)
$$

with $c_{f}$ as in 9). This implies, according to (126, the second limit in 125).

Case b: $-\frac{\pi}{2}<\varphi^{0}<0$ and $\varphi^{\mathbf{1}}=\mathbf{0}$. If $\bar{\sigma}=0$, the solutions such that $\varphi(0)=\varphi^{0}, \varphi\left(\tau_{\text {fin }}\right)=\varphi^{1}$ must be in the region $E$, and the proof from case (a) applies, where $\varphi^{1}$ is replaced with zero. If $\bar{\sigma}>0$, the solutions on the stable manifolds $\left\{\psi=S^{0}(\varphi)\right\}$ and $\left\{\psi=S^{\pi}(\varphi)\right\}$ (see Figure 7) also qualify for this case, because $\varphi$ reaches zero in finite time. Hence we have to examine the solutions such that $(\psi(0), \varphi(0))=\left(\chi, \varphi^{0}\right)$ with $\chi \in\left[S^{0}\left(\varphi^{0}\right), S^{\pi}\left(\varphi^{0}\right)\right]$ instead of the open interval; the solutions $\left(\psi^{\chi}(),. \varphi^{\chi}().\right)$ to the Cauchy problem (54)(122) are still well defined and depend continuously on $\chi$; however, uniqueness of $\tau_{\text {fin }}^{\chi}$ such that $(123)$ holds for $\chi \in\left(S^{0}\left(\varphi^{0}\right), S^{\pi}\left(\varphi^{0}\right)\right)$ but not for $\chi=S^{0}\left(\varphi^{0}\right)$ or $\chi=S^{\pi}\left(\varphi^{0}\right)$. If we call $\tau_{\text {fin }}^{S^{0}\left(\varphi^{0}\right)}$ (resp. $\tau_{\text {fin }}^{S^{\pi}\left(\varphi^{0}\right)}$ ) the first time $t$ such that $\varphi^{S^{0}\left(\varphi^{0}\right)}(t)=0$ (resp. $\varphi^{S^{\pi}\left(\varphi^{0}\right)}(t)=0$ ), the solutions to be considered are these with initial condition $(\psi(0), \varphi(0))=\left(\chi, \varphi^{0}\right), S^{0}\left(\varphi^{0}\right)<\chi<S^{\pi}\left(\varphi^{0}\right)$ on the time interval $\left[0, \tau_{\text {fin }}^{\chi}\right]$ and these with initial condition $(\psi(0), \varphi(0))=\left(\chi, \varphi^{0}\right), \chi \in\left\{S^{0}\left(\varphi^{0}\right), S^{\pi}\left(\varphi^{0}\right)\right\}$ on the time intervals $[0, \tau], \tau_{\text {fin }}^{\chi} \leq \tau<+\infty$. With the first set of solutions, one reaches $\bar{\lambda} \in\left[\Lambda\left(S^{0}\left(\varphi^{0}\right)\right), \Lambda\left(S^{\pi}\left(\varphi^{0}\right)\right)\right]$; the set of solutions with initial condition $\left(S^{0}\left(\varphi^{0}\right), \varphi^{0}\right)$ allows one to reach $\bar{\lambda}$ larger than $\Lambda\left(S^{0}\left(\varphi^{0}\right)\right)$ as the value of $\int_{0}^{\tau} c\left(\psi^{S^{0}\left(\varphi^{0}\right)}(s), \varphi^{S^{0}\left(\varphi^{0}\right)}(s)\right) \mathrm{d} s$ varies from $\Lambda\left(S^{0}\left(\varphi^{0}\right)\right)$ to $+\infty$ as $\tau$ varies from $\tau^{S^{0}\left(\varphi^{0}\right)}$ (the smallest such value such that $\left.\varphi S^{0}\left(\varphi^{0}\right)(\tau)=0\right)$ to $+\infty$; these solutions with initial condition $\left(S^{\pi}\left(\varphi^{0}\right), \varphi^{0}\right)$ allow one to reach $\bar{\lambda}$ smaller than $\Lambda\left(S^{\pi}\left(\varphi^{0}\right)\right)$.

Case c: $\varphi^{0}=\varphi^{1}=0$. It suffices to chose $\tau_{\text {fin }}=|\bar{\lambda}|$ and the solution to be the equilibrium $(0,0)$ if $\bar{\lambda} \geq 0$ or the equilibrium $(\pi, 0)$ if $\bar{\lambda} \geq 0$. Then (67) is satisfied because $c(0,0)=1, c(\pi, 0)=-1$.

Case d: $0<\varphi^{0}<\varphi^{1}<\frac{\pi}{2}$. This is the other "generic" case, with case (a). According to Lemma 2 , the solutions such that $\varphi(0)=\varphi^{0}, \varphi\left(\tau_{\text {fin }}\right)=\varphi^{1}$ must lie in one of the regions $F, E$, or $F^{+}$or in the unstable manifolds $\mathcal{U}^{0}$ 
or $\mathcal{U}^{\pi}$ that separate them (see Figure 77). These solutions satisfy $\varphi(0)=\varphi^{0}$, hence $S^{0}\left(\varphi^{0}\right)<\psi(0)<S^{\pi}\left(\varphi^{0}\right)$. For any $\chi$ in the open interval $\left(S^{0}\left(\varphi^{0}\right), S^{\pi}\left(\varphi^{0}\right)\right)$, let $\left(\psi^{\chi}(),. \varphi^{\chi}().\right)$ be the solution to the Cauchy problem (54)-(122). According to Lemma2.

- If $S^{0}\left(\varphi^{0}\right)<\chi<Z_{b}^{0}\left(\varphi^{0}\right)$ or $Z_{b}^{0}\left(\varphi^{\pi}\right)<\chi<S^{\pi}\left(\varphi^{0}\right), t \mapsto \varphi^{\chi}(t)$ is first decreasing, then crosses the set of zeroes of $b$ at some time $t^{o}: \psi^{\chi}\left(t^{o}\right)=Z_{b}^{0}\left(\varphi^{\chi}\left(t^{o}\right)\right)$ or $\psi^{\chi}\left(t^{o}\right)=Z_{b}^{\pi}\left(\varphi^{\chi}\left(t^{o}\right)\right)$ and is increasing for $t$ larger than $t^{o}$. Hence, since $\varphi^{\chi}\left(t^{o}\right)<\varphi^{0}<\varphi^{1}$, there is a unique $\tau_{\text {fin }}^{\chi}$ (larger than $\left.t^{o}\right)$ such that $\varphi^{\chi}\left(\tau_{\text {fin }}^{\chi}\right)=\varphi^{1}$.

- If $Z_{b}^{0}\left(\varphi^{0}\right) \leq \chi \leq Z_{b}^{\pi}\left(\varphi^{0}\right), t \mapsto \varphi^{\chi}(t)$ is monotonic increasing for positive times and cannot have a limit, hence it takes all the values between $\varphi^{0}$ and $\frac{\pi}{2}$ only once and there is a unique $\tau_{\text {fin }}^{\chi}$ such that $(123)$ holds.

In both cases, $Z_{b}^{0}\left(\varphi^{1}\right)<\psi^{\chi}\left(\tau_{\text {fin }}^{\chi}\right)<Z_{b}^{\pi}\left(\varphi^{1}\right)$, hence $b\left(\widehat{\varphi}^{\chi}\left(\tau_{\text {fin }}^{\chi}\right), \widehat{\psi}^{\chi}\left(\tau_{\text {fin }}^{\chi}\right)\right)>0$, and so there is a constant $k>0$ such that $\left|\varphi^{\chi}\left(t^{\prime}\right)-\varphi^{\chi}\left(t^{\prime \prime}\right)\right|>k\left|t^{\prime}-t^{\prime \prime}\right|$ for $t^{\prime}, t^{\prime \prime}$ in a neighborhood of $\tau_{\text {fin }}^{\chi}$. This implies that $\tau_{\text {fin }}^{\chi}$ depends continuously on $\chi$. This continuous dependence on $\chi$ allows us to define the continuous map $\Lambda:\left(U^{0}\left(\varphi^{0}\right), U^{\pi}\left(\varphi^{0}\right)\right) \rightarrow \mathbb{R}$ by (124); as in case (a), let us prove the following limits, sufficient to imply that $\Lambda$ is onto:

$$
\lim _{\substack{\chi \rightarrow U^{0}\left(\varphi^{0}\right) \\ \chi>U^{0}\left(\varphi^{0}\right)}} \Lambda(\chi)=+\infty, \lim _{\substack{\chi \rightarrow U^{\pi}\left(\varphi^{0}\right) \\ \chi<U^{\pi}\left(\varphi^{0}\right)}} \Lambda(\chi)=-\infty
$$

This follows as in case (a): first we get

$$
\lim _{\substack{\chi \rightarrow U^{0}\left(\varphi^{0}\right) \\ \chi>S^{0}\left(\varphi^{0}\right)}} \tau_{\text {fin }}^{\chi}=\lim _{\substack{\chi \rightarrow U^{\pi}\left(\varphi^{0}\right) \\ \chi<S^{\pi}\left(\varphi^{0}\right)}} \tau_{\text {fin }}^{\chi}=+\infty
$$

and then, with $f=\left|\varphi_{1}\right|$, 127] holds for $\chi$ close to $U^{0}\left(\varphi^{0}\right)$ and (128) for $\chi$ close to $U^{\pi}\left(\varphi^{0}\right)$.

Case e: $0<\varphi^{0}=\varphi^{1}<\frac{\pi}{2}$. This is a similar to the previous case but degenerate in the sense that $\tau_{\text {fin }}^{\chi}=0$ if $Z_{b}^{0}\left(\varphi^{0}\right) \leq \chi \leq Z_{b}^{\pi}\left(\varphi^{0}\right)$. The only nontrivial trajectories that display the same initial and final values of $\varphi$ lie in the regions $F$ or $F^{+}$, and they join points on one side of the curve where $b$ vanishes $\left(\psi=Z_{b}^{0}(\varphi)\right.$ or $\left.\psi=Z_{b}^{\pi}(\varphi)\right)$ to points on the other side. We have

$$
\Lambda(\chi) \begin{cases}>0 & \text { if } U^{0}\left(\varphi^{1}\right)<\chi<Z_{b}^{0}\left(\varphi^{1}\right), \\ =0 & \text { if } Z_{b}^{0}\left(\varphi^{1}\right) \leq \chi \leq Z_{b}^{\pi}\left(\varphi^{1}\right), \\ <0 & \text { if } Z_{b}^{\pi}\left(\varphi^{1}\right)<\chi<U^{\pi}\left(\varphi^{1}\right)\end{cases}
$$

(129) still holds and the same arguments prove that $\Lambda$ is onto.

\section{References}

1. Arnold, V.I.: Mathematical methods of classical mechanics, Graduate Texts in Mathematics, vol. 60, 2nd edn. Springer-Verlag, New York (1989).

2. Bao, D., Chern, S.S., Shen, Z.: An introduction to Riemann-Finsler geometry, Graduate Texts in Mathematics, vol. 200. Springer-Verlag, New York (2000)

3. Bombrun, A., Pomet, J.B.: The averaged control system of fast oscillating control systems. SIAM J. Control Optim. 51(3), 2280-2305 (2013). DOI 10.1137/11085791X.

4. Bonnard, B., Caillau, J.B.: Geodesic flow of the averaged controlled Kepler equation. Forum Mathematicum 21(5), $797-814$ (2009). DOI 10.1515/FORUM.2009.038.

5. Bonnard, B., Caillau, J.B., Dujol, R.: Energy minimization of single input orbit transfer by averaging and continuation. Bull. Sci. Math. 130(8), 707-719 (2006)

6. Bonnard, B., Faubourg, L., Trélat, E.: Mécanique céleste et contrôle des véhicules spatiaux, Mathématiques \& Applications, vol. 51. Springer-Verlag, Berlin (2006)

7. Bonnard, B., Sugny, D.: Optimal Control with Applications in Space and Quantum Dynamics, AIMS Series on Applied Mathematics, vol. 5. AIMS (2012)

8. Edelbaum, T.N.: Optimum low-thrust rendezvous and station keeping. AIAA J. 2, 1196-1201 (1964)

9. Edelbaum, T.N.: Optimum power-limited orbit transfer in strong gravity fields. AIAA J. 3, 921-925 (1965)

10. Filippov, A.F.: Differential equations with discontinuous righthand sides, Mathematics and its Applications (Soviet Series), vol. 18. Kluwer Academic Publishers Group, Dordrecht (1988). DOI 10.1007/978-94-015-7793-9.

11. Geffroy, S.: Généralisation des techniques de moyennation en contrôle optimal - Application aux problèmes de rendez-vous orbitaux en poussée faible. Thèse de doctorat, Institut National Polytechnique de Toulouse, France (1997)

12. Geffroy, S., Epenoy, R.: Optimal low-thrust transfers with constraints-generalization of averaging technics. Acta Astronautica 41(3), 133-149 (1997). DOI 10.1016/S0094-5765(97)00208-7.

13. Hartman, P.: Ordinary Differential Equations, 2 edn. Birkhäuser (1982)

14. Hirsch, M.W., Smale, S., Devaney, R.L.: Differential Equations, Dynamical Systems, and an Introduction to Chaos. Academic Press (2004)

15. Pontryagin, L.S., Boltyanskii, V.G., Gamkrelidze, R.V., Mishchenko, E.F.: The mathematical theory of optimal processes. Interscience Publishers John Wiley \& Sons, Inc. New York-London (1962) 